\title{
Noise Characterization of a Full-Scale Nose Landing Gear
}

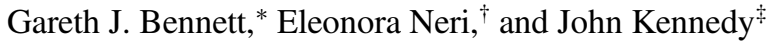 \\ Trinity College Dublin, University of Dublin, D02 PN40 Dublin, Republic of Ireland \\ DOI: $10.2514 / 1 . C 034750$
}

\begin{abstract}
This paper presents experimental results from a nose landing-gear test campaign. A highly detailed model of the nose section of a 90-seat configuration green regional aircraft concept was built and tested in an aeroacoustic open-jet wind tunnel at full scale. All of the landing-gear components, fixtures, and small details and associated structures such as the complete wheel bay, bay doors, and hydraulic dressings were included at full scale. This paper focuses on one element of the testing: that of component noise assessment. The dressings, wheels, torque link, steering pinion, bay doors, and then the main strut and drag stay were removed in succession, and an acoustic evaluation was performed at a range of velocities from $M=0.12$ to $M=0.19$. In addition, hub caps were installed on the wheels, and their performance as a low-noise treatment is presented and assessed for efficacy. The landing gear, doors, and bay generated most noise in the frequency range between 120 and $400 \mathrm{~Hz}$, but appreciable noise above the baseline was measured up to $10 \mathrm{kHz}$. The low- to midfrequency aerodynamic noise was found to scale with $V^{6}$, as usual; however, the spectra at high frequencies collapsed perfectly when scaled to $V^{7}$, confirming the fact that high-frequency noise is radiated from the turbulent flow surrounding small features. The total noise output of the landing-gear assembly differs from that of the sum of the components in isolation due to installation effects. The research in this paper studied these effects and the influence of the components on each other.
\end{abstract}

Nomenclature

$\begin{array}{ll}B & =\text { open bay fuselage } \\ D & =\text { doors } \\ \text { Dress } & =\text { Dressings } \\ F & =\text { closed fuselage } \\ L & =\text { main strut with drag stay and wheel axle (no dressings) } \\ N & =\text { nose } \\ S & =\text { steering pinion } \\ T & =\text { torque link } \\ W & =\text { wheels } \\ x & =\text { streamwise coordinate (equal to } 0 \text { at nozzle exit plane) } \\ y & =\text { spanwise coordinate (equal to } 0 \text { at test section } \\ z & =\text { height coordinate (equal to } 0 \text { on test section floor) }\end{array}$

\section{Introduction}

A IRCRAFT noise has become critical in aviation design because it has a negative impact on the health of people living in the vicinity of airports $[1,2]$. The total noise generated by aircraft is from the engines and the airframe, with the latter being a result of unsteady flow over the aircraft structure, in particular, the high-lift devices such as flaps, slats, and landing gear. Research efforts to date have produced impressive results. The modern commercial aircraft is 75\% more fuel-efficient per passenger kilometer than the first generation of jet aircraft, and with respect to noise, there has been a $20 \mathrm{~dB}$ reduction because of the widespread introduction of the commercial jet aircraft in the 1960s [3] . Examination of this downward trend in the acoustic output shows that, although there have been steady improvements made over time due to incremental advances, it has been the implementation of innovative technology solutions related to engine design and bypass ratio that has resulted in the biggest stepchange reductions.

Received 15 September 2017; revision received 4 May 2018; accepted for publication 20 May 2018; published online Open Access 6 August 2018. Copyright $\odot 2018$ by Gareth J. Bennett. Published by the American Institute of Aeronautics and Astronautics, Inc., with permission. All requests for copying and permission to reprint should be submitted to CCC at www.copyright.com; employ the ISSN 0021-8669 (print) or 1533-3868 (online) to initiate your request. See also AIAA Rights and Permissions www.aiaa.org/randp

*Associate Professor, School of Engineering. Senior Member AIAA.

${ }^{\dagger}$ Graduate Student, School of Engineering.

${ }^{\ddagger}$ Research Fellow, School of Engineering.
With these significant reductions in the principal sources of aeroengine noise (the jet and the tonal contributions from the fan, compressor, and turbine), a new noise floor has been reached that is related to the broadband noise contributions of the engine and, most significantly in the context of this paper, airframe noise. During aircraft approach to landing, when engines are operating at low thrust, the noise from the landing gear and the wheel bay cavity contributes substantially and can often be a major contributor to the overall noise signature of modern aircraft. Specifically, of the total aircraft noise, that radiated from the landing gear varies for short-range to longrange aircraft from 31 to $40 \%$, which compares significantly when compared to that from the engine, which ranges from 38 to $42 \%$ [4]. Because landing gear is typically deployed at the $1500 \mathrm{ft}$ altitude position on the 3 deg glide slope, radiated noise disturbs communities for many kilometers outside the airport boundary.

Landing gear is mechanically complex, primarily designed to support the load of a landing aircraft but also required to kinematically retract into the fuselage after takeoff to reduce drag. Its design, as a priority, is constrained by requirements associated with safety, inspection, and maintenance. This has resulted in a large number of components clustered together of very different sizes, shapes, and orientations. The air flow over these components causes unsteady surface pressures and the resulting aerodynamic noise is treated as being analogous to that from dipole sources, with the acoustic intensity being proportional to velocity to the sixth power [5].

Many experimental campaigns have been conducted to characterize landing-gear noise. Seminal studies in the 1970s provided initial insight [6], with later campaigns, enabled by the availability of larger test facilities, showing that full-scale and more detailed models are required to capture the high-frequency contribution of small components [], $]$, which hitherto had been thought to be unimportant. Results from such tests indicate that landing gear, for the most part, generates broadband noise caused by the interaction of the landing gear with turbulent flow. In addition, distinct narrowband noise contributions can arise from coherent periodic vortex shedding from cylindrical components, and significant levels of tonal noise have been measured from cavity resonance due to, for example, uncapped pin holes [9]. The frequency range for these contributions is typically up to $3 \mathrm{kHz}$ [10] which overlaps with the most sensitive range of human noise perception and emphasizes the importance of landing-gear noise reduction.

As an alternative approach to wind-tunnel testing, a direct measure of landing-gear noise, and indeed the assessment of applied lownoise treatments [11], has been attempted by source localization techniques and flyover measurements using microphone arrays [12]. 
This approach is excellent in principle because it measures the actual production landing gear in final installation conditions and environment. Such measurements enable relative magnitudes to be determined and unexpected tones to be localized to landing gear as the source region $[13,14]$. However, these measurement campaigns are extremely expensive to conduct, and although they are satisfactory for the verification of final design improvements, they are not cost-effective for the execution of parametric analysis. In addition, given the distance from the arrays to the landing gear, the low spatial resolution does not allow for analysis on a component basis nor does the aperture allow for low-frequency analysis. Challenges also arise from different and louder noise sources overlapping the frequency range of interest, although tests have been performed with aircraft engines operating at minimum idle reduced thrust $(N 1=20 \%)[11]$ to increase the signal-to-noise ratio.

Based on experimental test campaigns, various semi-empirical schemes to predict landing-gear noise have been conceived. These methods are beneficial due to their speed and simplicity to implement. Fink [15] devised one of the first techniques, developing an empirical method based on both flight-test data and simplified scale-model wind-tunnel tests. This led to a standard on aircraft noise prediction developed at NASA: the Aircraft Noise Prediction Program (ANOPP). However, because these experimental data were also based on simplified models, an early limitation of ANOPP (since updated) was that it tended to underpredict high-frequency components. Subsequently, Smith and Chow [16] as well as Guo [17] and Guo et al. [18] incorporated high-frequency noise into their models using Airbus and Boeing data sets. Smith and Chow examined the landing gear on a component level including the highfrequency noise generated from the small detail with a "dressing factor". Guo's method distinguishes between three increasing sizes of gear components responsible for low-, medium-, and highfrequency noise contributions. High-frequency noise generated by small construction details is treated with a "complexity factor". However, Guo's scheme does not provide a direct measure of the contribution of each individual gear element to the overall noise. Lopes et al. [19] proposed to address this with the Landing Gear Model and Acoustic Prediction framework. Within this framework, the landing gear is viewed as an assembly of fundamental acoustic elements, such as cylinders, wheels, or trailing edges. Unfortunately, because the preceding methods rely on empirical inputs, they have limited reliability in predicting the impact of add-on noise reduction devices or new or unconventional designs.

Because of increasing computational capabilities, computational aeroacoustics (CAA) now provides the most promise in allowing highfidelity results to be obtained at reasonable costs. Hybrid methods, combining, for example, detached-eddy simulation [20] with the Ffowcs Williams-Hawkings analogy [21], allow realistic landing-gear models to be numerically modeled $[22,23]$, and entirely different approaches using lattice Boltzmann methods [24] seem to be able to dramatically decrease computational times, allowing larger and larger models to be analyzed. However, these numerical methods and others are still in development and need to be validated against experimental results provided by dedicated datasets such as the Benchmark Problems for Airframe Noise Computations initiative [25].

This paper presents experimental results from the Clean Sky funded ALLEGRA "Advanced Low Noise Landing (Main and Nose) Gear for Regional Aircraft" project. This project was developed to assess low-noise technologies applied to a full-scale nose landinggear model and a half-scale main landing-gear model of a 90-seat configuration regional aircraft concept. This Green Regional Aircraft concept (see Fig. 1) was developed as an integrated technology demonstrator (ITD) to allow technologies that best suit the environmental goals set for regional aircraft that will enter service from 2020 onward to be matured and validated.

As has been discussed in this section, source localization techniques from flyover measurements are expensive to conduct and provide limited detail of noise sources. In addition, whereas semiempirical methods and CAA have significant benefits, they require detailed experimental results to inform and validate them. With regard to the nose landing gear (NLG) campaign, one of the significant contributions of ALLEGRA is that a complete and highly detailed representation of the landing-gear components and associated structures such as the complete wheel bay cavity (wheel well), bay doors, nose fuselage and hydraulic dressings were included at full scale. To the authors' knowledge, this is the first time that all of these aspects, at full scale, have been examined in an experimental campaign.

This paper will focus on one element of the NLG testing: that of component removal assessment. The dressings, wheels, torque link, steering pinion, bay doors, and then the main leg and drag stay were removed in succession (see Fig. 2), and an acoustic acquisition was performed at a range of velocities. The open, empty bay was also closed over so that its contribution in isolation could also be assessed. Such an approach to component removal assessment has precedent with a limited number of components (primarily low-noise treatments) being removed in succession within the SILENCER test campaign [26] and by Guo et al. [27] where a simplified main landing-gear model with no wheel well (there was an open cavity) or doors was examined and wheels of different sizes and dressings were removed. It is important to note and expressed previously [26] that the removal of components in this manner does not necessarily identify the contribution of that component in isolation as might be determined by a numerical model, where wheels, for example, can be effectively suspended in space and exposed to a simulated free flow. The landing gear, being a cluster of components, is subject to a mean incident flowfield, but it is the position of those components relative to each other and the bay, doors, and fuselage that controls local flowfields and therefore the associated noise generation. For example, the leg-torque link interaction noise is affected by the close proximity of the wheels, and similarly the interwheel noise could be modified by their close presence in turn. Physical surfaces can also reflect and shield sound. However, it is this very behavior of how components can suppress or amplify the noise generation of the components they neighbor that this study examines. The open bay, as another example, has been demonstrated to radiate significant tonal

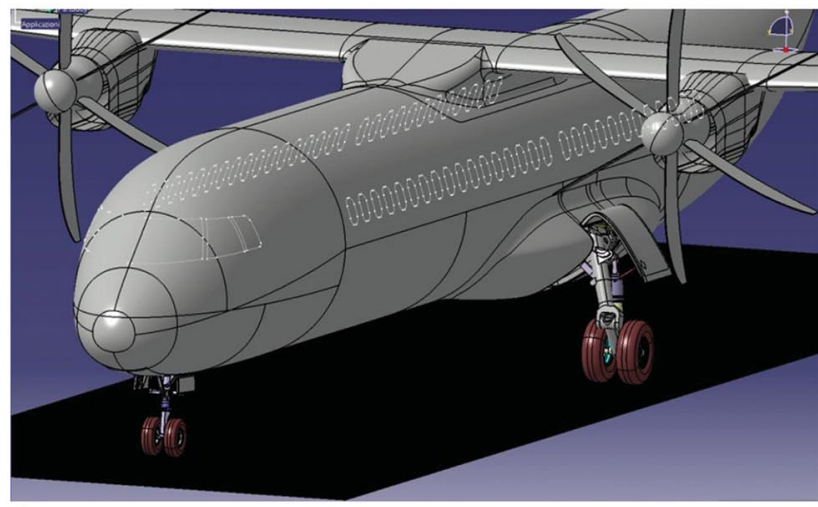

a)

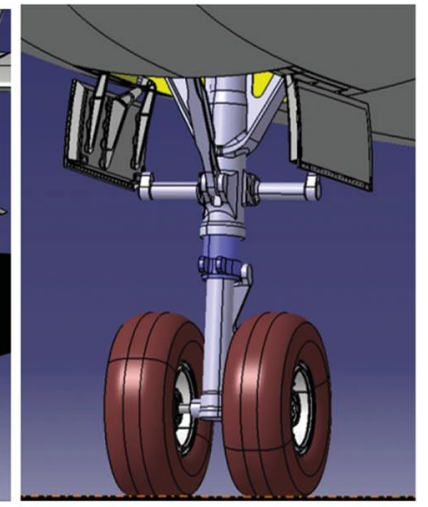

b)

Fig. 1 Clean Sky Green Regional Aircraft ITD 90-seat Aircraft configuration concept. 


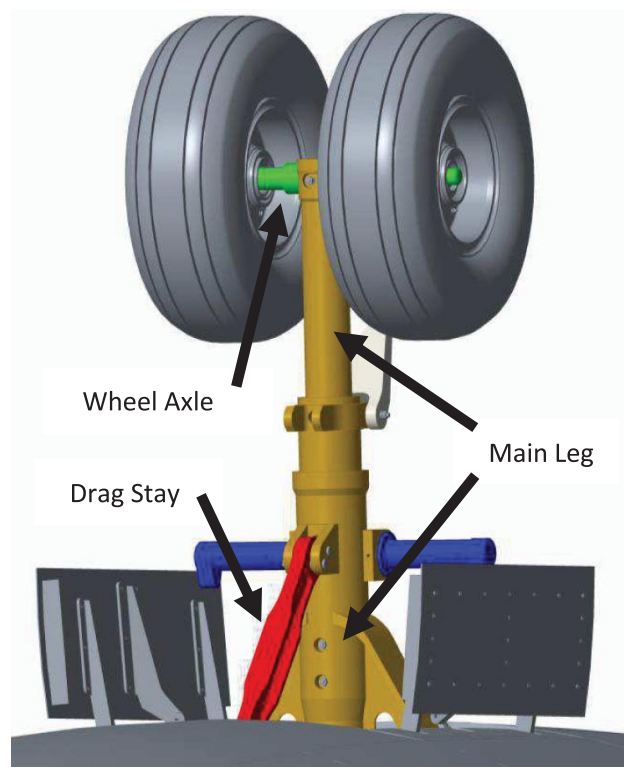

a) View from upstream

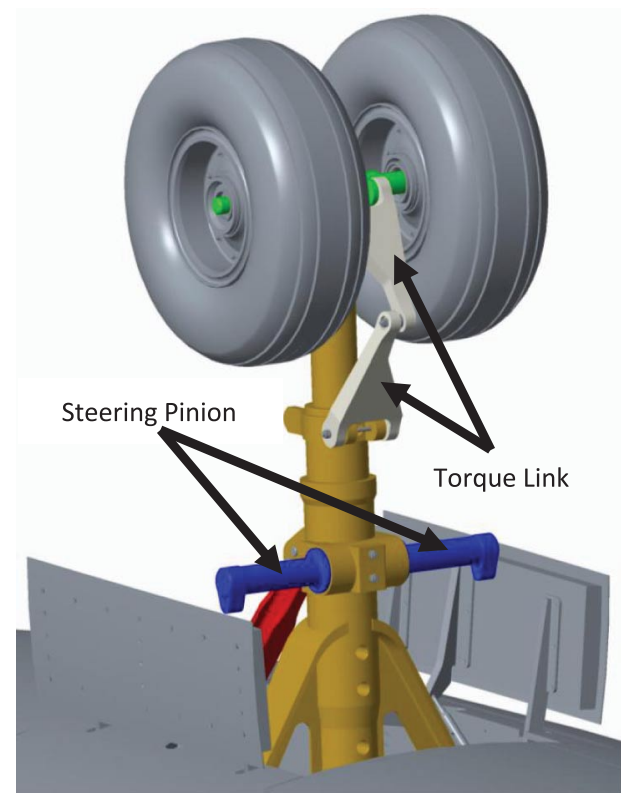

b) View from downstream

Fig. 2 Nose landing gear (NLG) components.

noise at its Helmholtz frequency and first two standing wave duct resonance tones in the velocity range of a landing aircraft [28], with equivalent tones found for the main landing gear (MLG) test setup, even with the leg in situ [29]. Such tones or high internal pressure amplitudes would be problematic for weapons bays or for passengers in vehicles with open windows, but we see in this study that the presence of the doors and main leg is sufficient to disrupt the shear layer so that these tones are no longer excited. Issues associated with the influence of components on each other are discussed in detail in this paper.

\section{Experimental Setup}

\section{A. Wind Tunnel, Instrumentation, and Landing-Gear Model}

Tests were performed in the Pininfarina open-jet aeroacoustic wind-tunnel facility in Turin, Italy (see Fig. 3), which has an acoustically treated test section of $8 \times 9.60 \times 4 . \overline{2} 0 \mathrm{~m}$. The facility contains a main downstream fan of 29 blades operating at a low rotation frequency and an additional semicircular array of 13 fans in the return circuit to increase flow velocities and decrease noise in the test section. The maximum flow speed achievable in the empty test section is $72 \mathrm{~m} / \mathrm{s}$, where the semicircular nozzle area is $11 \mathrm{~m}^{2}$. The velocity produced by the wind tunnel is very uniform, varying by only $0.5 \%$ over the area. The turbulence level can be adjusted up to $8 \%$, but the default of $0.3 \%$ was used in these tests.

Figure 4 illustrates the relative position of the nose landing-gear model in the wind tunnel as well as the coordinate system employed, where the $x-z$ plane through the origin is the symmetry plane of the test model, the $y-z$ plane through the origin corresponds to the windtunnel nozzle exit plane, and the origin is situated on the floor of the testing platform. The full-scale nose landing-gear model complete with the nose fuselage and bay cavity and doors was positioned in the wind tunnel so that the distance between the wind-tunnel nozzle exit plane and the landing-gear wheel axis was $2.8 \mathrm{~m}$. The wheel axle midpoint coordinates were

$$
X=2.8 \mathrm{~m}, \quad Y=0 \mathrm{~m}, \quad Z=2.175 \mathrm{~m}
$$

A number of microphone arrays were installed inside the test section and used in the test campaign. Some of these can be seen in the photos in this paper. In addition, surface pressure sensors were installed in the bay and on the landing gear. However, this work will focus on the measurements using the far-field linear array of 13 microphones indicated by black circles in Fig. 4. Unlike the MLG, the NLG is considered to have some acoustic directionality [6]. There is some clearance between the fuselage and wheel that is dominated in form by the vertical cylindrical shape of the main strut. The array was positioned in a straight line out of the flow at a height of $z=2.72 \mathrm{~m}$ from the wind-tunnel floor and at $y=-3.46 \mathrm{~m}$ from the wind-tunnel streamwise axis. This provided a good compromise location to allow a combination of the sideline influence and some indication of sources radiated to the flyover direction to be measured. A single microphone from the top beamforming array, directly above the wheel axle, will also be used in this paper when an exact flyover measurement is required. The distribution of the microphones in the far-field array was chosen to cover the maximum emission angle possible spanning angles from approximately 41 to 142 deg relative to the nose landing-gear wheel axle midpoint. An angle of $0 \mathrm{deg}$ would indicate upstream. These angles are calculated in the plane intersecting the straight line of microphones and the midpoint of the wheel axle. These are geometric angles, and no correction has been made here to account for flow effects such as refraction by the shear

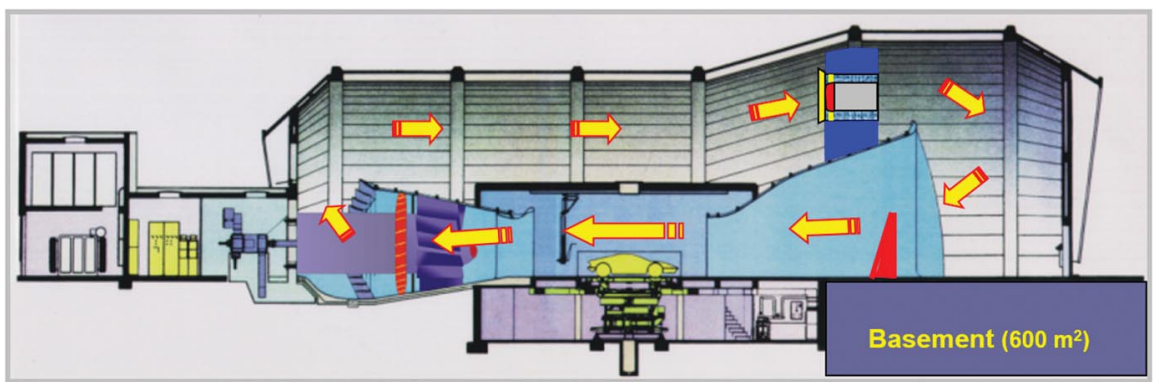

Fig. 3 Pininfarina aeroacoustic open-jet wind tunnel. 


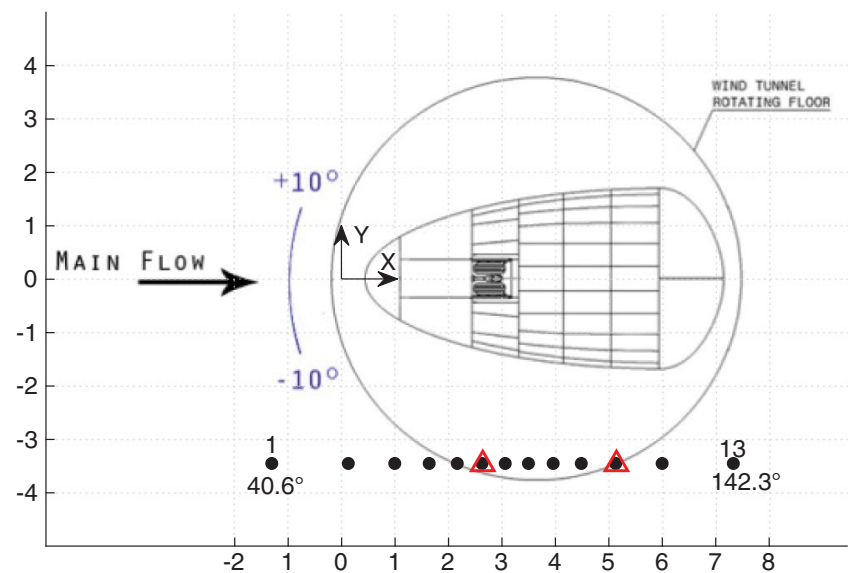

Fig. 4 Schematic of NLG model in wind tunnel frame of reference. Linear far field array microphones indicated with black circles. Microphones 6 and 11 are highlighted with triangles.

layer. This is primarily due to the deliverable specification of the ALLEGA project, which requested data in this form. It is the understanding of the consortium that this raw form of data would subsequently be entered into numerical codes for validation and that shear-layer corrections would be treated at that stage. Data were acquired for $10 \mathrm{~s}$ at a sampling rate of $32,768 \mathrm{~Hz}$.

\section{B. Test Configurations}

The configurations of component removal reported in this paper are summarized in Table 1, and photographs of the different stages of the decomposition are shown in Figs. $\underline{5}$ and $\underline{6}$. The reader is again directed to Fig. 2 for component descriptions. The dressings are not shown in the $\overline{C A D}$ files of Fig. 2 but can be seen (with some difficulty) in Fig. $\underline{5 a}$. In this design of NLG, there are very few dressings and fixtures required. The reader's attention is directed to two lengths of blue tubing running along either side the drag stay and over the steering pinion in addition to two lengths of white tubing running along the steering pinion.

In Table 1 , the test IDs for the configurations tested during the disassembly of the nose landing gear are formed through subtraction of the term in the nomenclature corresponding to the component that is removed from the NLG, which represents the fully dressed nose landing gear installed in the bay of the fuselage complete with doors. The NLG configuration is considered to be the baseline test.

The photos show the manufactured fuselage, which includes the actual nose section. As noted repeatedly in previous test campaigns, although the actual flow velocity just upstream of the MLG can be $20 \%$ lower than the freestream velocity due to installation effects and circulation resulting from lift and its location under the wing [7,27], the NLG is exposed to higher velocities than that of the freestream due to the acceleration under the nose. A significant differentiator between this test campaign and previous ones is that the relative velocity and flowfield at the NLG is accurate relative to the freestream velocity. Also evident in the photos is the level of detail

Table 1 Test configurations

\begin{tabular}{lcc}
\hline \hline Test ID & Fuselage & Landing gear \\
\hline NLG & Open & Dressed \\
NLG-Dress & Open & Undressed \\
NLG-DressW & Open & NLG-Dress with wheels removed \\
NLG-DressWT & Open & NLG-Dress with wheels and torque link \\
& & removed \\
NLG-DressWTS & Open & NLG-Dress with wheels, torque link and \\
& & steering pinion removed \\
NLG-DressWTSD & Open & NLG-Dress with wheels, torque link, \\
& & steering pinion and doors removed \\
NB & Open & None \\
NF & Sealed & None \\
\hline \hline
\end{tabular}

and very accurate representation of the production NLG, where even representations of the door hinges are included.

Each model configuration was tested at a variety of flow speeds: $40 \mathrm{~m} / \mathrm{s}(M=0.12), 50 \mathrm{~m} / \mathrm{s}(M=0.15), 60 \mathrm{~m} / \mathrm{s}(M=0.17)$, and $65 \mathrm{~m} / \mathrm{s}(M=0.19)$ and yaw settings ranging between $\pm 10 \mathrm{deg}$, $M=0.2$ would be the typical operational Mach number value at approach conditions for this aircraft. The yaw settings allowed the performance of the technology to be evaluated under conditions equivalent to landing with a crosswind; however, this paper only presents results for the 0 deg yaw angle. In addition, a fixed "angle of attack" of 4 deg was built into the model. Obviously, the relative velocity vector as seen by the NLG is determined in reality by its angle relative to the plane's horizontal axis, the plane's horizontal axis relative to the ground, the plane's net approach angle, the velocity vector of any significant wind, and their velocity magnitudes. The angle of $4 \mathrm{deg}$ was provided by the Clean Sky ITD Topic Manager LEONARDO and remained fixed for these tests.

\section{Results}

\section{A. Nose Landing-Gear Noise}

Preliminary tests were conducted to characterize the background noise. No acoustic measurements were recorded of the empty wind tunnel; therefore, the spectra of the NF configuration characterizes both the sealed bay fuselage and the wind-tunnel acoustic output. The NF configuration, therefore, is considered to be the baseline background noise case, above which we can identify the contributions from individual components and assemblies such as the wheel bay, doors, landing gear, etc. One of the additional benefits of manufacturing this accurate nose fuselage in the test campaign, as well as to house the wheel bay, was to provide the correct boundary layer and aerodynamic flowfield, resulting from the associated curvatures, to impinge upon the gear.

Narrow and $1 / 3$ octave band A-weighted spectra were calculated using the linear far-field array of microphones. Most spectra are confined to the region between $100 \mathrm{~Hz}$ and $10 \mathrm{kHz}$, where the main noise sources are found, although an examination below $100 \mathrm{~Hz}$ will be presented when discussing the wheel bay noise.

Figure 7 a presents a comparison between the NLG and the NF configurations, at microphone 6 , as a function of velocity, and therefore an indication of the noise generated by the landing gear, bay, and doors, above the NF configuration noise level, is readily available. The results are processed in $1 / 3$ octave band scale, which is common practice, but which is also beneficial here because the frequency averaging effect produces a cleaner curve at high frequencies, which allows the difference between NLG and NF to be more easily determined. Examining the $40 \mathrm{~m} / \mathrm{s}$ velocity initially, it can be seen that the NLG components contribute significant noise above the background, NF, noise level. The contribution is greatest (over $3 \mathrm{~dB}$ ) up to approximately the $1.6 \mathrm{kHz} 1 / 3$ octave band center frequency, but it is significant to see that a measurable contribution is recorded up to the $10 \mathrm{kHz}$ center frequency. The general trend of the delta between the NF and NLG configurations is quite consistent, with increasing velocity indicating that both NF and NLG are attributable to aerodynamic sources, although there are peaks below $500 \mathrm{~Hz}$, which require closer scrutiny with narrowband analysis. Figure $7 \mathrm{~b}$ provides this narrowband analysis for the NLG case. To note here is that, up to approximately $1.25 \mathrm{kHz}$, the spectra are characterized by narrowband peaks that remain consistent in frequency with increasing velocity. In addition, in the same figure, there are a couple of tones that increase in frequency with velocity. Tones at $125 \mathrm{~Hz}$ and its first two harmonics, which occasionally appear in the analysis, are associated with the wind-tunnel Blade Pass Frequency (BPF) and are to be disregarded. However, other tones exist that, in principle, could be caused by vortex shedding from cylindrical components in the landing gear. This research attempts to identify which components of the NLG these noise sources are associated with.

Given that a range of velocities was examined, an amplitude scaling was conducted. As discussed in Sec. I, it is generally agreed that the aerodynamic noise generated by landing gear is a result of dipole-type pressure fluctuations due to unsteady pressure forces. 


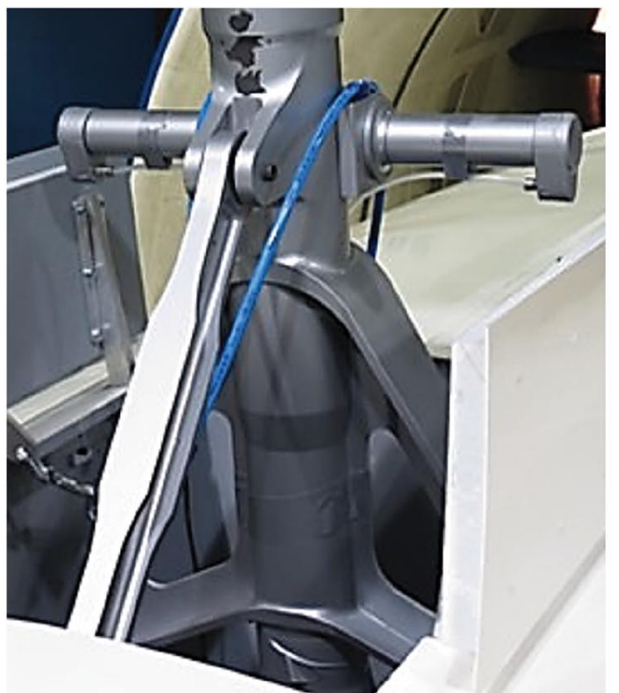

a) NLG

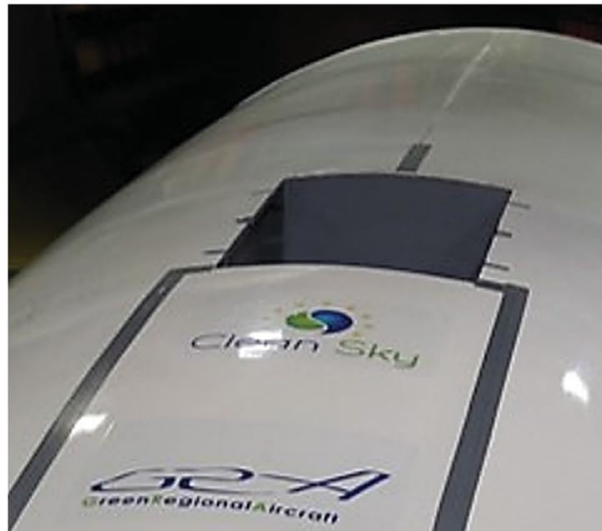

c) $\mathrm{NB}$

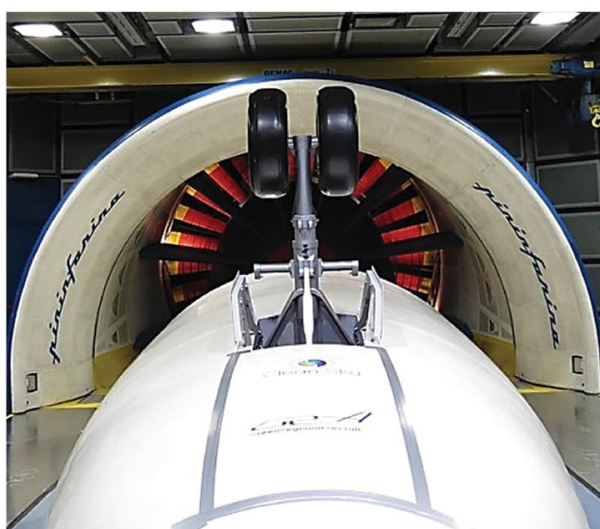

b) NLG-Dress

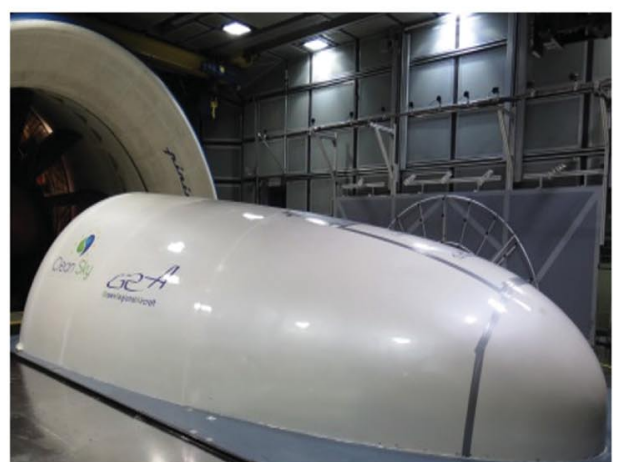

d) $\mathbf{N F}$

Fig. 5 Test configurations A. Photos of the model at different stages of component removal.

The theory of Curle [5] shows that, in such instances, the acoustic intensity or pressure squared term is proportional to $V^{6}$. For the ALLEGRA data, $V^{6}$ proportionality was confirmed when beamforming analysis from the top (flyover) array was compared to outdoor flyover beamforming analysis of real aircraft of similar design [14]. Figure 7c shows a very satisfactory collapse of sound levels when scaled in this manner. However, this theory only holds true when the sound sources are compact. That is to say, the relationship is true only when the geometric size of the sound source is small when compared to the acoustic wavelength. Guo et al. [27], for example, demonstrate that, for small details in landing gear, in their case the dressings and fixtures, this assumption is no longer valid. The small details generate high-frequency noise, and therefore the wavelength is no longer large compared to the noise sources and can no longer be considered to be compact. Instead of surface pressure fluctuations, they discuss that the noise sources are instead radiated from the turbulent flow that surrounds the small features of the landing gear, and therefore velocity requires a scaling exponent of a higher order. Reviewing Fig. 7c once more, we see that the collapse is excellent for low to midfrequencies, with the exception of some tones (to be discussed later), but at higher frequencies, above approximately $1.2 \mathrm{kHz}$, the amplitude still seems to increase with velocity. Figure $7 \mathrm{~d}$ shows the curves scaled, this time to velocity to the seventh power, and above $1.2 \mathrm{kHz}$ there seems to be a perfect collapse. Given that Fig. 7a shows appreciable NLG noise up to $10 \mathrm{kHz}$, and that this noise scales to $V^{7}$, we can conclude that it is the small features of the NLG model that are producing this noise. Given the high level of detail of the manufactured NLG model, it is not just the additional dressings and fixtures, as in Guo et al., that are contributing to this high-frequency noise, but all the small features to be found such as the hinges, wheel hub detail, fixtures, rods, ribs, pins, gussets, etc.

Figure 8 shows the NF spectra subtracted from the NLG spectra, in A-weighted decibels, for each of the microphones in the array in the form of a position versus frequency: spectrogram plot. The spectra are narrowband, and only the $0 \mathrm{~Hz}$ to $2 \mathrm{kHz}$ range is examined. By using the full array, we capture some sense of directivity of the NLG as a cluster, but also, for this and subsequent such plots, we maximize the chance of measuring noise from sources/components with specific directivity or which may be shielded by other components. Unfortunately, microphone 12 seemed to be faulty in some of these tests and has been excluded from the spectrogram plots. Given that we have examined the behavior of the noise as a function of velocity in Fig. 7, we chose to present only the $50 \mathrm{~m} / \mathrm{s}$ velocity level from this point on.

The NLG configuration, consisting of the landing gear, doors, and wheel bay, increases the noise at these microphone locations by up to approximately $12 \mathrm{~dB}$ above the baseline background noise level across a wide frequency range, especially in the region of 200 and $350 \mathrm{~Hz}$, where the radiation direction tends to favor upstream. The noise depicted in this spectrogram includes any installation effects and reflections from the fuselage, and although no local velocity measurements were taken, it can be assumed that the NLG is exposed to the correct accelerated velocity over the nose and the correct associated boundary layer as discussed earlier.

\section{B. Contribution of Individual Landing-Gear Components}

Regarding the disassembly of the nose landing gear, six components were progressively removed as outlined previously: dressings, wheels, torque link, steering pinion, doors, and the leg. In 


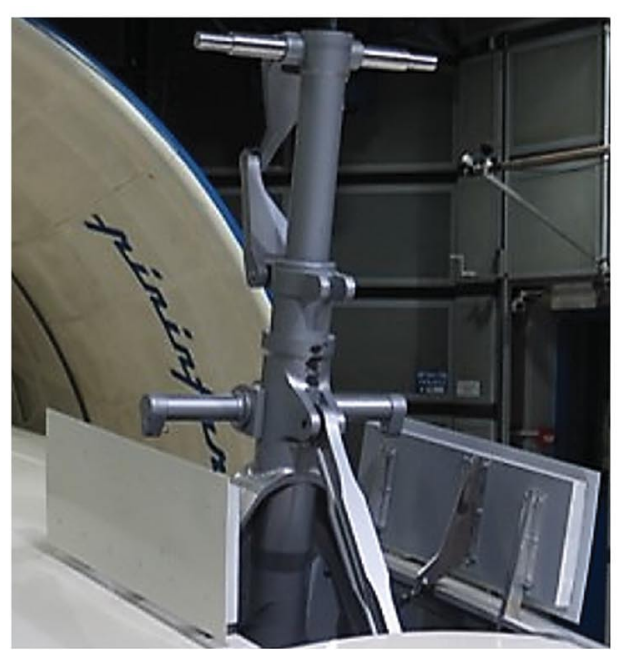

a) NLG-DressW

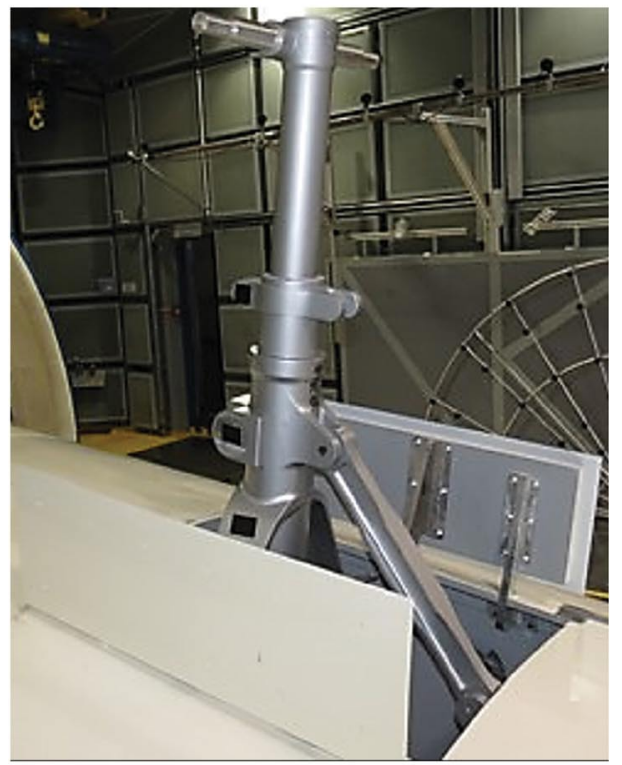

c) NLG-DressWTS

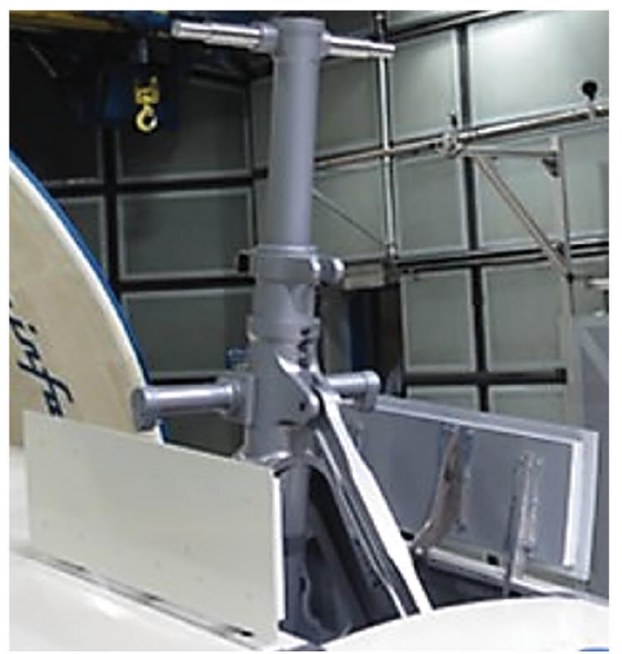

b) NLG-DressWT

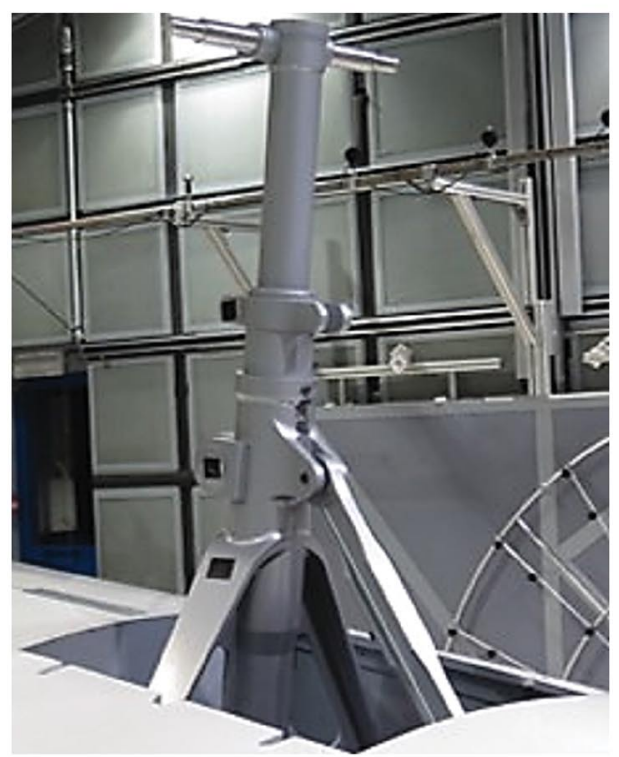

d) NLG-DressWTSD

Fig. 6 Test configurations B. Photos of the model at different stages of component removal.

this section, spectrograms as in Fig. 8 will be calculated to assess the effect of a component to the overall noise change. They will be presented as a delta, where the difference in the noise level before and after the removal of a component is calculated. It is of interest to examine the noise level difference due to the removal of these components and, equally, the effect they have on noise sources through their addition. If the noise level after the component is removed is subtracted from the noise level before it is removed, then the spectrogram can be interpreted as the net effect of adding the component to the assembly. Therefore, a negative value (colored blue online) can be interpreted as a noise source that has been suppressed by the addition of the component, and a positive value (colored red online) is noise caused by the addition of the component, whether directly or indirectly. Because of a lack of time, only one sequence could be performed. Ideally, the order of removal could have been changed with additional tests. In particular, the result of removing the torque link before the wheels and the leg before the doors would have yielded additional information. Additionally, the removal of the wheel axle and the drag stay would also have been informative.

The contribution of the dressings was minor for the NLG, and it is not reported in this paper because it was found to be of the order of only $\pm 1 \mathrm{~dB}$. Interestingly, a slight decrease in noise level due to the presence of the dressings was found at some emission angles. This is thought to be due to the beneficial effect of the dressings disrupting the formation and shedding of vortices from cylindrical components. A greater contribution from the dressings was found for the MLG model, and this point will be discussed in future publications examining the main landing gear.

\section{Effect of the Wheels: NLG-DressW}

The spectrogram in Fig. 9 presents the difference in noise level between NLG-Dress and NLG-DressW. That is to say, the result when the noise level of the configuration with the wheels removed (NLG-DressW) is subtracted from the configuration that has the wheels still attached (NLG-Dress). High-amplitude levels in the spectrogram indicate where, in space and in frequency, the addition of the wheels has increased the noise. Examining the spectrum first for reductions in levels to see if any NLG-DressW sound sources have been suppressed by the addition of the wheels, we see initially some distinct blue lines at approximately 125 and $250 \mathrm{~Hz}$. These, upon close inspection, lie immediately beside straight red lines, all of which are to be disregarded. These are the wind-tunnel BPF tone and first harmonic. Differences in loading in the tunnel seem to have had an effect on the BPF frequencies so that, when the spectrograms are calculated through subtraction, due to the slight frequency shift, the two peaks do not cancel out but instead result in a high positive peak and negative dip remaining. The reader is directed to Neri et al. [28] for further discussion. There is, however, an additional lower 


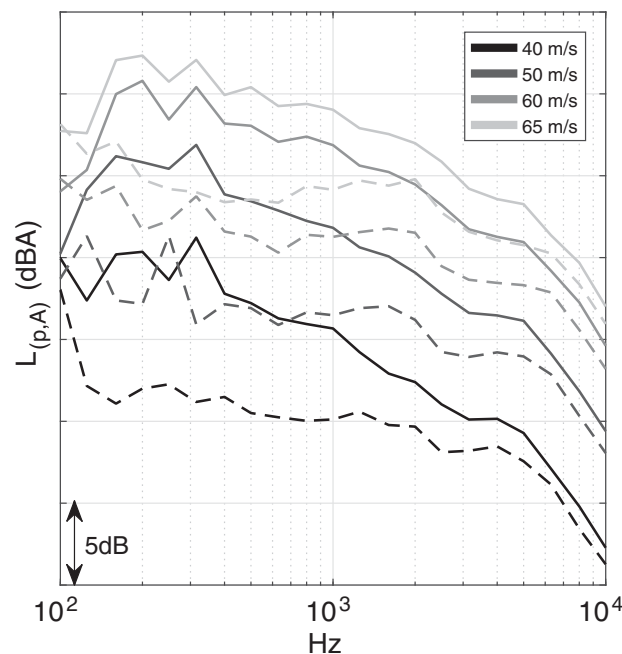

a) NLG SPL (solid line) and NF SPL (dashed line). $1 / 3$ octave band

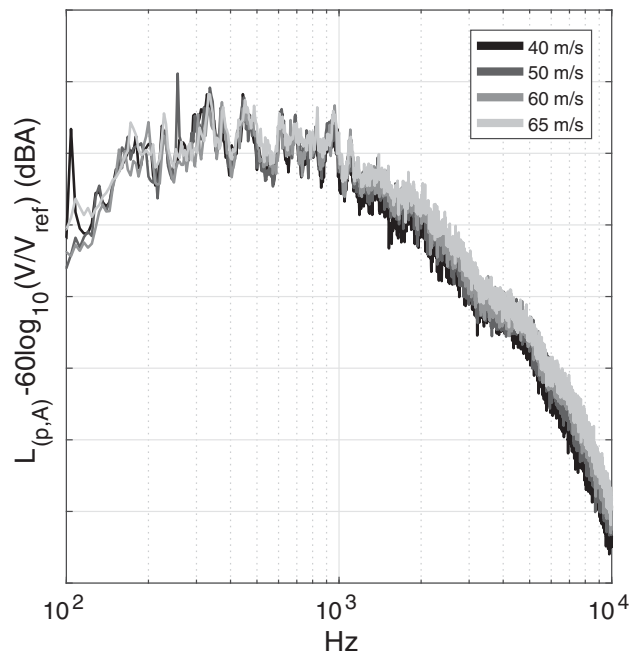

c) NLG scaled $V^{6}$

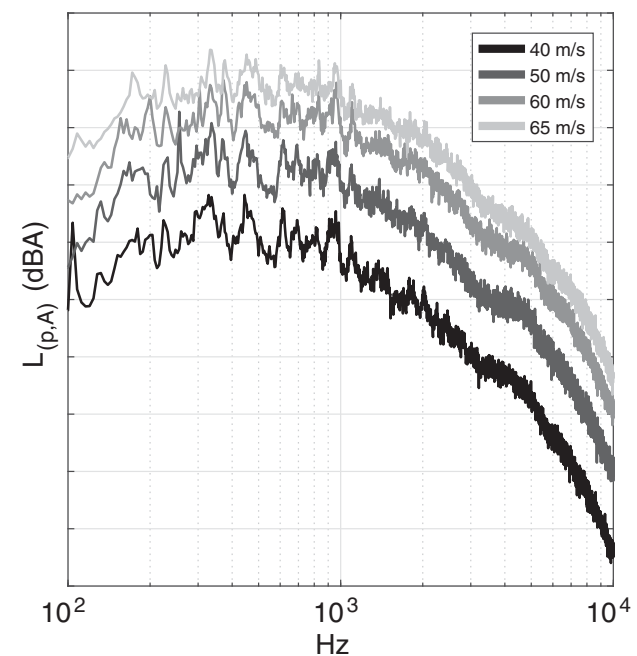

b) NLG SPL. Narrowband

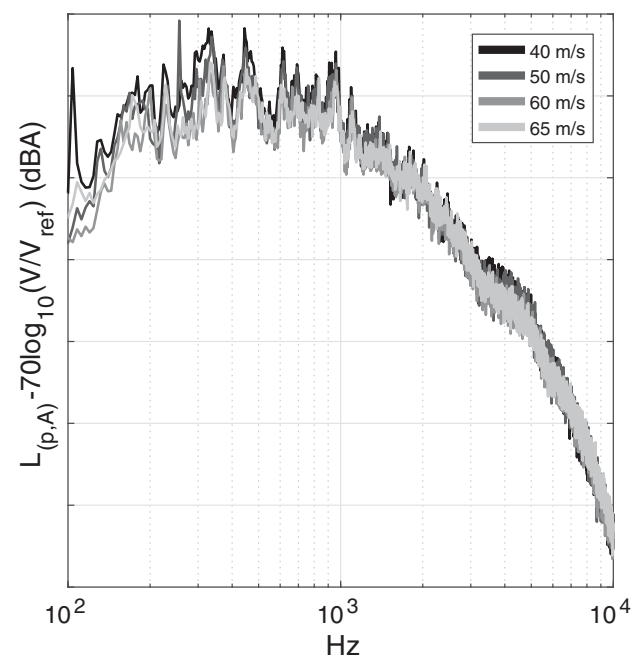

d) NLG scaled $V^{7}$

Fig. 7 NLG and NF configurations as a function of tunnel velocity at the microphone 6 position.

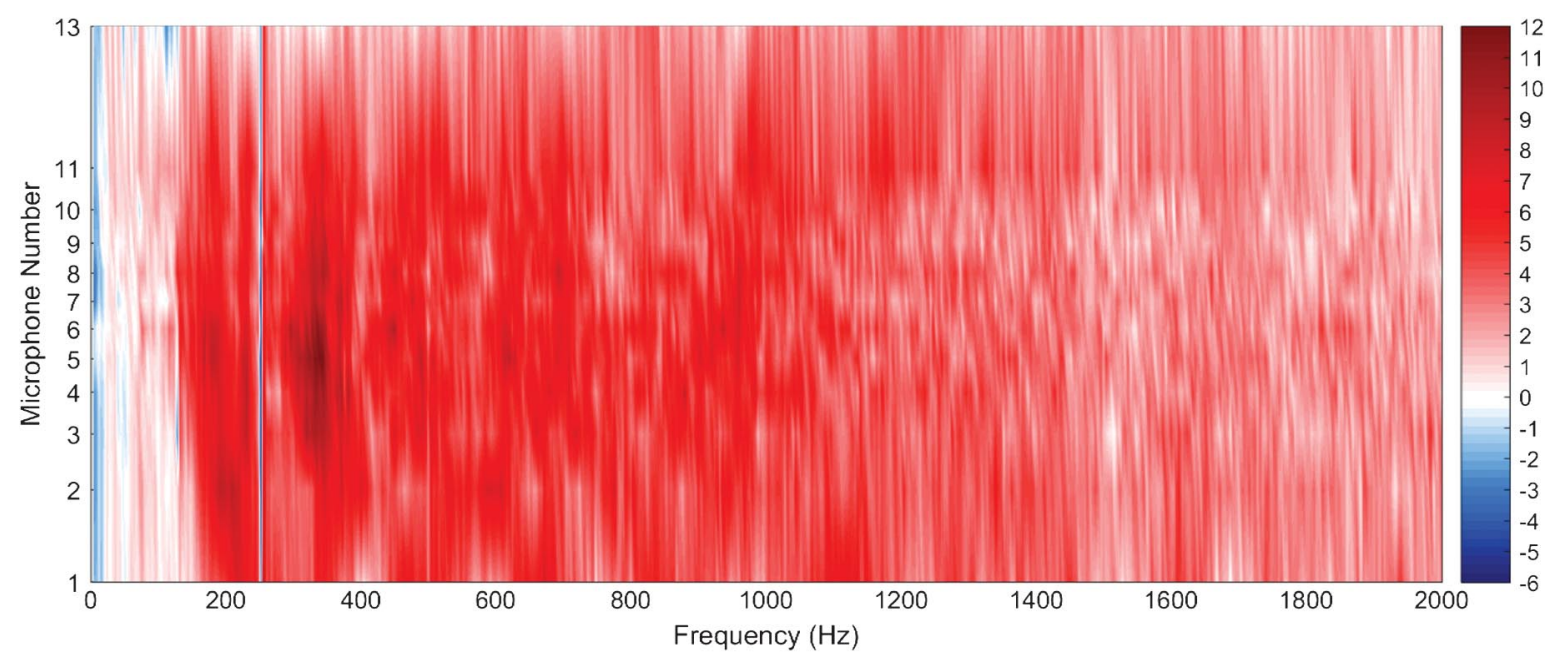

Fig. 8 (NLG)-(NF) A-weighted $\triangle$ SPL. Acoustic signature from dressed nose landing gear, doors, and wheel bay once the fuselage and wind-tunnel noise have been subtracted. $50 \mathrm{~m} / \mathrm{s}$.

amplitude reduction at $200 \mathrm{~Hz}$, which covers a wide range of angles. Given its tonal nature, it is potentially the result of vortex shedding from one of the cylindrical components. Table 2 compiles the dimensions of some of the likely components. In addition to the diameters, the Reynolds numbers for $50 \mathrm{~m} / \mathrm{s}$ and the vortex shedding frequency are provided. It is very difficult to provide accurate Strouhal numbers because the length-to-diameter ratios of some of the components are relatively short; they typically terminate at a 
Table 2 Component characteristics and vortex shedding frequencies

\begin{tabular}{lcccc}
\hline \hline Item & Diameter, $\mathrm{m}$ & $\mathrm{Re}$ & $S t$ & Frequency, $\mathrm{Hz}$ \\
\hline Wheel axle & 0.04 & $1.2 \times 10^{5}$ & 0.2 & 250 \\
Main strut & 0.08 & $2.4 \times 10^{5}$ & 0.22 & 138 \\
Main strut & 0.11 & $3.3 \times 10^{5}$ & 0.38 (smooth) or 0.23 (rough) & 173 or 105 \\
Main strut & 0.12 & $3.8 \times 10^{5}$ & 0.5 (smooth) or 0.23 (rough) & 208 or 89 \\
Steering pinion & 0.05 & $1.5 \times 10^{5}$ & 0.2 & 200 \\
\hline \hline
\end{tabular}

junction with another component at one end, whereas at the other, they do not, and the surface roughness is difficult to estimate. The surface finish of the painted machined aluminum is good, but the components are not perfect cylinders. The larger-diameter section of the main strut, for example, spreads into side stays. In addition, the Reynolds numbers are quite high, near or exceeding the critical limit. Despite this, some estimates are provided using the results of Achenbach and Heinecke [30] to provide the Strouhal numbers, particularly for the smooth versus rough cylinders at high Reynolds number. From the table, it seems that the addition of the wheels suppresses vortex shedding from the steering pinion at $200 \mathrm{~Hz}$. There is less than a wheel diameter between them, and so it is conceivable that the addition of the wheels disrupts the flow over the steering pinion sufficiently to prevent shedding. Given the direction of the local flow and the wheels' location, it is unlikely that the presence of the wheels has a significant effect on the bay, drag stay, doors, or fuselage, although comparison with CFD/CAA would be useful to verify this.

Examining now the increase in noise through the addition of the wheels, there is certainly a significant increase: up to $8 \mathrm{~dB}$ over a wide frequency range up to $1.2 \mathrm{kHz}$. A particularly concentrated increase in the 260 to $400 \mathrm{~Hz}$ range and around $1 \mathrm{kHz}$ and $650 \mathrm{~Hz}$ is noticeable. Referring to the literature, and in particular the output from the LAGOON program "Landing Gear Noise Database for CAA Validation" [31], an interesting finding has been the prediction of tonal peaks away from the flyover plane, which are due to acoustic modes being excited in the circular rim cavities of the two facing wheels. More precisely, the shear-layer instability modes couple with acoustic resonant modes, which are responsible for side noise radiation and side force oscillations. Our far-field linear array is well placed to detect such tones. A numerical study of these noise sources was performed by Casalino et al. [32] using a lattice Boltzmann method. They examined the interwheel area and found that the shear layer that forms over the wheel rims excites acoustic depth and azimuthal modes between and within the rims, which can result in acoustic radiation to the far field. The mechanism is similar to shear-layer excited cylindrical cavities [33-35]. Casalino et al. [32] define two lengths between the wheels. The first is the "floor-to-floor rim distance", or $l_{\mathrm{FF}}$, which is the distance between the bottom of the rim cavity of one wheel and that of the other. The second is the "edge-to-edge rim distance", or $l_{\mathrm{EE}}$, which is taken here to be the closest distance between the wheel tires. The shear layers excite half-wavelength-depth modes between these surfaces. In the ALLEGRA NLG, these lengths are approximately $l_{\mathrm{FF}}=0.33 \mathrm{~m}$ and $l_{\mathrm{EE}}=0.16 \mathrm{~m}$. Using the correction factors of 0.82 and 1.11 determined by Marsden et al. [36] and Casalino et al. [32], which account for the geometrical differences between the wheel assembly and a pure annular cavity in a flat plate, frequencies of $f_{\mathrm{FF}}=344 /(2 * 0.33 * 0.82)=$ $636 \mathrm{~Hz}$ and $f_{\mathrm{EE}}=344 /(2 * 0.16 * 1.11)=968 \mathrm{~Hz}$ are determined. These values are very close indeed to frequencies where high levels of noise are measured in Fig. 9. Casalino et al. also investigated azimuthal mode excitation in their numerical model, which remains to be done for the ALLEGRA data. Further investigation may allow other noise output as calculated in Fig $\underline{9}$ to be explained, but it is significant to note that these tones have been measured from full-scale representative landing gear, possibly for the first time.

\section{Effect of the Torque Link: NLG-DressWT}

With the wheels already detached, the torque link was then removed. The spectrogram in Fig. 10 shows the difference between NLG-DressW and NLG-DressWT. $\bar{A}$ though not as significant as the addition of the wheels, the addition of the torque link results in a number of high-amplitude peaks up to $4 \mathrm{~dB}$ greater than the NLGDressW configuration, one of particular interest at $148 \mathrm{~Hz}$, which is seen in many of the spectrograms. This might correspond to the vortex shedding frequency of the main strut, where the presence of the torque link has caused the vortex shedding to be amplified. Of additional interest is the reduction of noise radiated in the downstream direction across a wide frequency range. The torque link, it seems, increases noise in the upstream direction, whereas it suppresses noise from the bare main strut in the downstream direction. Referring back to the previous spectrogram, below $200 \mathrm{~Hz}$, this situation is then reversed when the wheels are added. The upstream radiated noise from the torque link is suppressed by the addition of the wheels as expected, given that the torque link is nestled between the wheels on the downstream side of the strut.

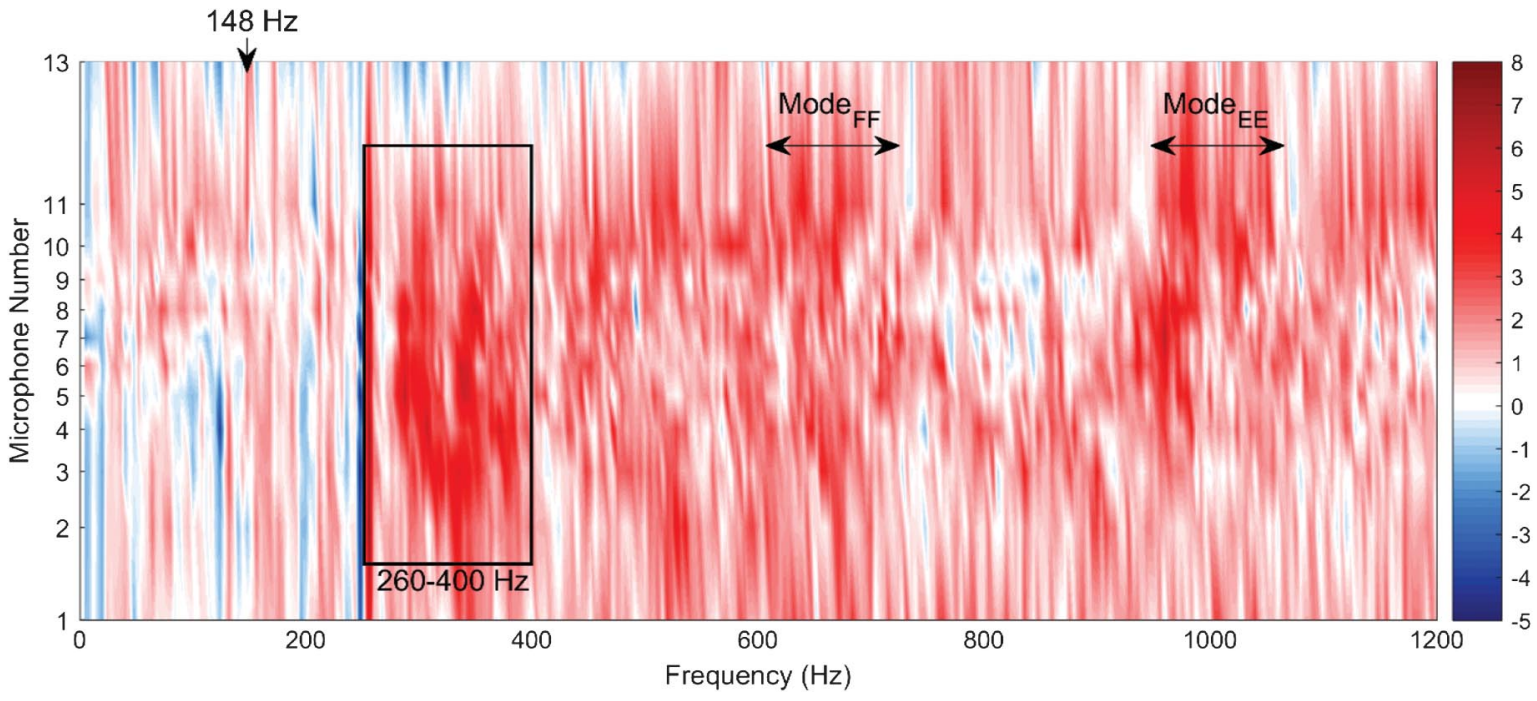

Fig. 9 (NLG-Dress)-(NLG-DressW) A-weighted $\Delta$ SPL. Effect of adding the wheels. $50 \mathrm{~m} / \mathrm{s}$. 


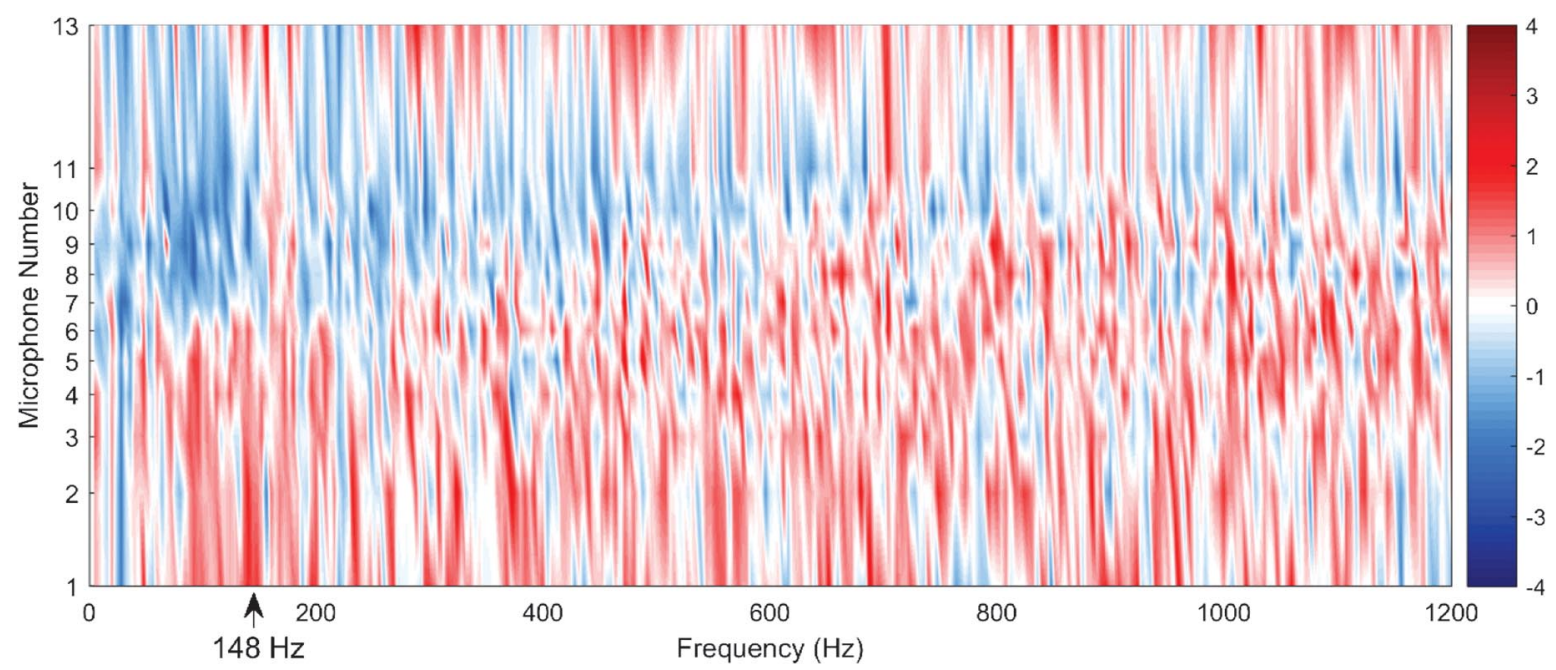

Fig. 10 (NLG-DressW)-(NLG-DressWT) A-weighted $\Delta$ SPL. Effect of adding the torque link. $50 \mathrm{~m} / \mathrm{s}$.

\section{Effect of the Steering Pinion: NLG-DressWTS}

The addition of the steering pinion, as shown in Fig. 11, results in two clear frequency bands of narrowband noise up to $4 \mathrm{~dB}$ in amplitude. One is to be seen at approximately $200 \mathrm{~Hz}$, which corresponds to its vortex shedding frequency (see Table 2) and seems to support the suggestion made earlier that the addition of the wheels suppresses this noise source. In addition, there are a number of highamplitude peaks that seem to occur at higher-order harmonics of this vortex shedding frequency. The second narrowband frequency band is around approximately $320 \mathrm{~Hz}$, which is not well understood but may be due to the steering pinion end caps to be seen in Fig. $\underline{6}$.

\section{Effect of the Doors: NLG-DressWTSD}

The addition of the doors results in a high-amplitude noise contribution over a wide range of frequencies: up to $9 \mathrm{~dB}$ from $120 \mathrm{~Hz}$ to $1.2 \mathrm{kHz}$, as shown in Fig. 12. There is a concentrated band of noise with a tendency to radiate in the upstream direction centered at $200 \mathrm{~Hz}$, which can be identified in Fig. 8. As can be seen in Fig. 6c, the geometric form of the hinges presents significant potential for noise generation.

The spectrogram also indicates a significant noise reduction, due to the installation of the doors, at 32 and $104 \mathrm{~Hz}$. This will be discussed in more detail in the next section.

5. Effect of Main Strut and Drag Stay on Wheel Well Noise

At this point in the component removal, only the main strut and the drag stay remain of the NLG within the bay. Figure $\underline{13}$ shows the
(NLG-DressWTSD)-(NB) spectrogram, and therefore it is a measure of the noise generated when the main strut and drag stay are added to the empty bay. From observation of the plot, it is clear that this assembly contributes significant noise, up to $8 \mathrm{~dB}$, over a wide range of frequencies up to $1.2 \mathrm{kHz}$, and through cross-referencing with Fig. 8 , is largely responsible for the noise in the 120 to $300 \mathrm{~Hz}$ frequency band. With the exception of a tone at approximately $148 \mathrm{~Hz}$, the majority of this noise radiates to the $90 \mathrm{deg}$ sideline or to the upstream direction, similar to the addition of the doors. The tone at $148 \mathrm{~Hz}$, having already been observed in a number of plots, especially when the torque link was added, corresponds almost certainly to the vortex shedding frequency of some self-determined average diameter of the main strut. As seen in Fig. 6d, the diameter of the strut is not consistent, it is inclined, and it still has the tow bar fixture and other irregularities along its length preventing it from being a perfect cylinder, but it does seem reasonable, given its length to (average) diameter ratio, that vortex shedding could result from its exposure to the mean flow. Referring to Table 2, a vortex shedding frequency of $148 \mathrm{~Hz}$ is possible. Reviewing the previous spectrograms, this tone is also observed when both the wheel and the torque link are added, highlighting the complex interrelationship between the components. The torque link, being aligned along the same axis as the strut, possibly behaves as a second tandem cylinder, energizing the vortex shedding. The addition of the wheels possibly concentrates the flow over the strut.

Given the location of the main strut in the bay and its exposure to the shear layer, which certainly must form from the leading edge of the bay, it is most likely that the narrowband noise in the 120 to

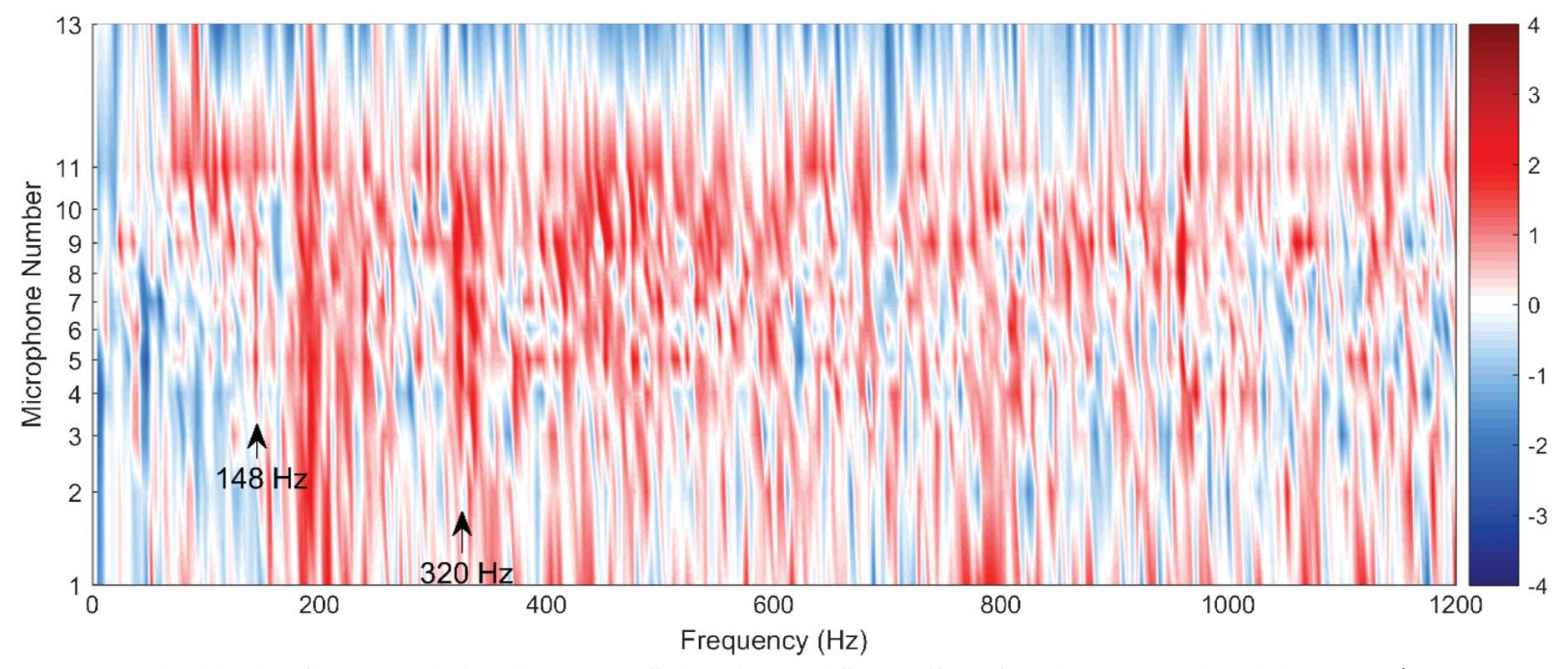

Fig. 11 (NLG-DressWT)-(NLG-DressWTS) A-weighted $\Delta$ SPL. Effect of adding the steering pinion. $50 \mathrm{~m} / \mathrm{s}$. 


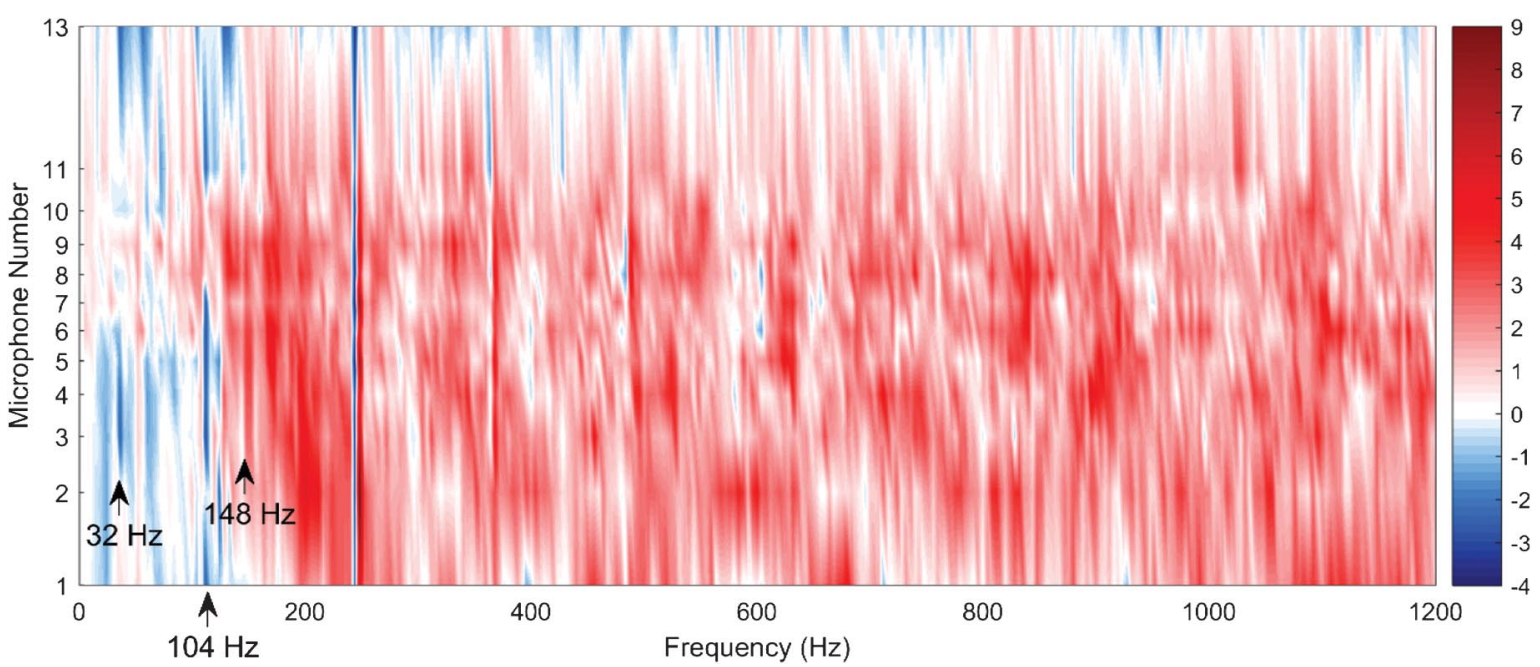

Fig. 12 (NLG-DressWTS)-(NLG-DressWTSD) A-weighted $\Delta$ SPL. Effect of adding the doors. $50 \mathrm{~m} / \mathrm{s}$.

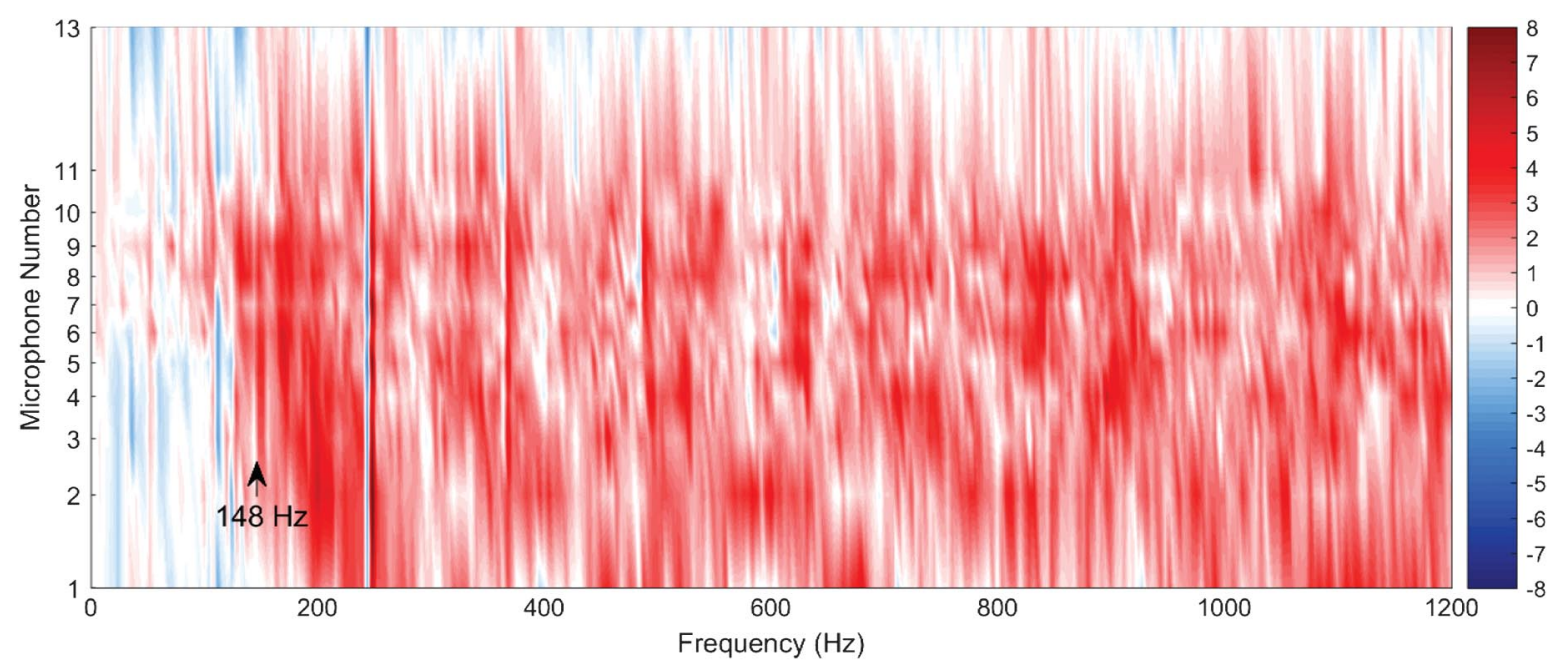

Fig. 13 (NLG-DressWTSD)-(NB) A-weighted $\triangle$ SPL. Effect of adding the main strut and drag stay. $50 \mathrm{~m} / \mathrm{s}$.

$300 \mathrm{~Hz}$ frequency band is a result of the shear layer impinging on the leading edge of the main strut.

Also to be seen in this spectrogram is the noise reduction of a tone at $32 \mathrm{~Hz}$ upon the addition of the main strut and drag stay. This reduction is related to the reduction we saw in the previous spectrogram at $104 \mathrm{~Hz}$ (also seen here) when the doors were added. These 32 and $104 \mathrm{~Hz}$ tones are caused by the Helmholtz resonance and the first resonant duct mode of the empty wheel bay. An extensive analysis of these tones and the noise-generating behavior of the wheel well was performed already for the ALLEGRA data by Neri et al. [28], and so only a few summary comments will be provided here along with an additional observation. The previous study found that Helmholtz resonance and high-amplitude cavity resonance of the first two duct modes of the wheel well can be measured by the farfield microphones for the NB configuration (i.e., when the bay is empty of the gear and the doors are removed). The noise-generating mechanisms of these tones were validated with a numerical study, and of significance, these tones were shown to be excitable within the velocity range of a landing aircraft. Once the gear and doors were added to the open bay (NB) configuration, the tonal noise no longer radiated. The cause of the suppression being explained by the disruption of the shear layer by the presence of the gear, which we see previously, causes instead the generation of noise in a different frequency range.

Figure 14 plots a single spectrum for the NB configuration for microphone 5 only at $50 \mathrm{~m} / \mathrm{s}$. In the plot, we see a clear measure of the Helmholtz resonance (HR in the plot) as well as the first and second duct tones. Of significant interest are sum and difference tones generated through interaction between the Helmholtz frequency and the first cavity tone, indicating that the sideline measurement is more than just the linear sum of individual sources but can also include nonlinear interactions between them. The background noise windtunnel tones WT1, WT2, and WT3, as discussed earlier, are also indicated.

In the spectrogram of Fig. 12, we saw that adding the doors significantly suppressed the $104 \overline{\mathrm{Hz}}$ duct tone and the $32 \mathrm{~Hz}$ tone. At first thought, one might think that this is due to the doors blocking the noise radiating to the far-field microphone array. However, this is not the case because the microphones are sufficiently high, and even for the upstream microphones, there is a reduction. The fact that the doors are suppressing the tones and not simply shielding them is confirmed in reference to Fig. 15, where the spectra with and without the addition of the doors are presented for a microphone from the top array, which is situated directly above the landing gear. It is evident from the plot that, with the suppression of the duct tone, the difference tone is also eliminated, as would be expected. See Bennett and Fitzpatrick [37] and Davis and Bennett [38] for such analysis.

An additional comment from this work is that, although in the paper by Neri et al. [28] it was concluded that the addition of the landing gear into the bay along with attaching the doors resulted in the suppression of the tonal noise, it seems, in fact, that it is the doors, in particular, that cause full suppression. The addition of the main strut and drag stay is sufficient to partially attenuate the Helmholtz resonance, but it requires the addition of the doors also to fully attenuate it and the first duct tone. This point is supported by reference to Fig. 16 , which is the spectrogram of the 


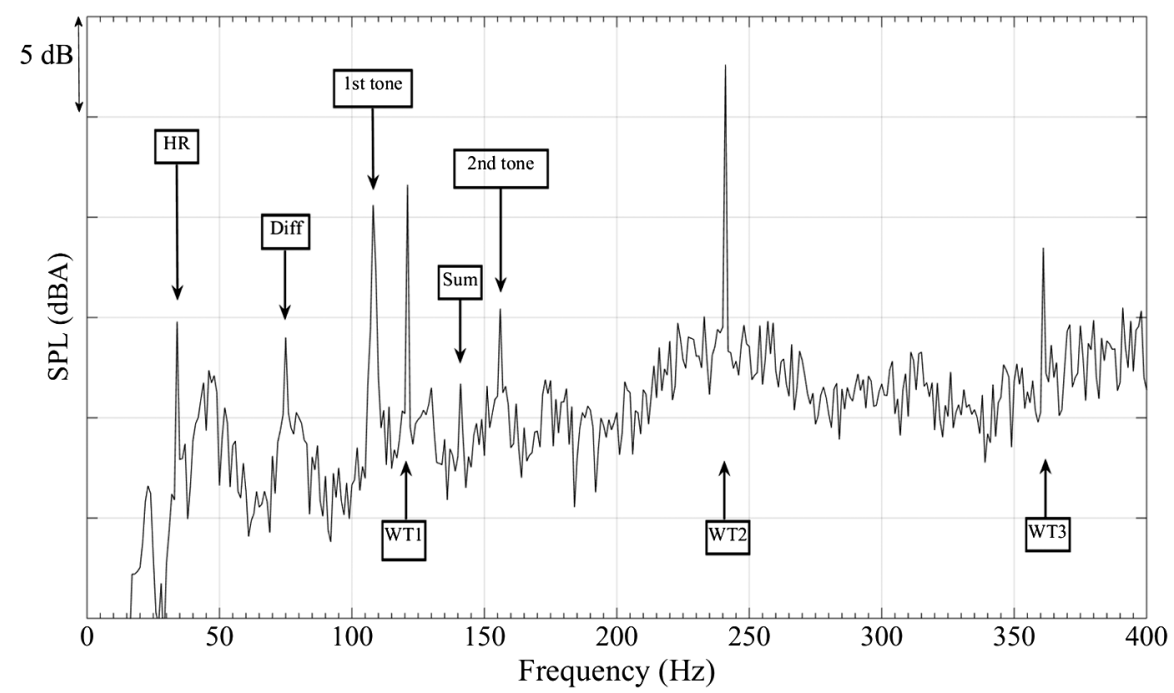

Fig. 14 Narrowband experimental A-weighted spectra for the NB configuration. $50 \mathrm{~m} / \mathrm{s}$, microphone 5 of the linear far-field array.

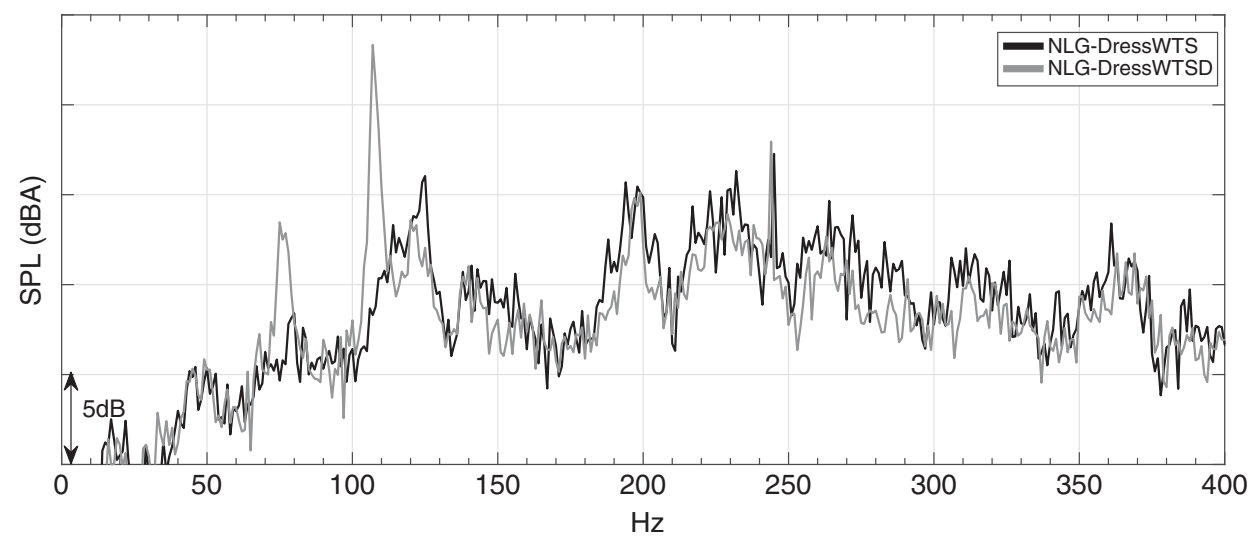

Fig. 15 Noise radiation to flyover position at a microphone directly above the wheel axle. Noise attenuation of the wheel bay duct tone though the addition of the bay doors is shown.

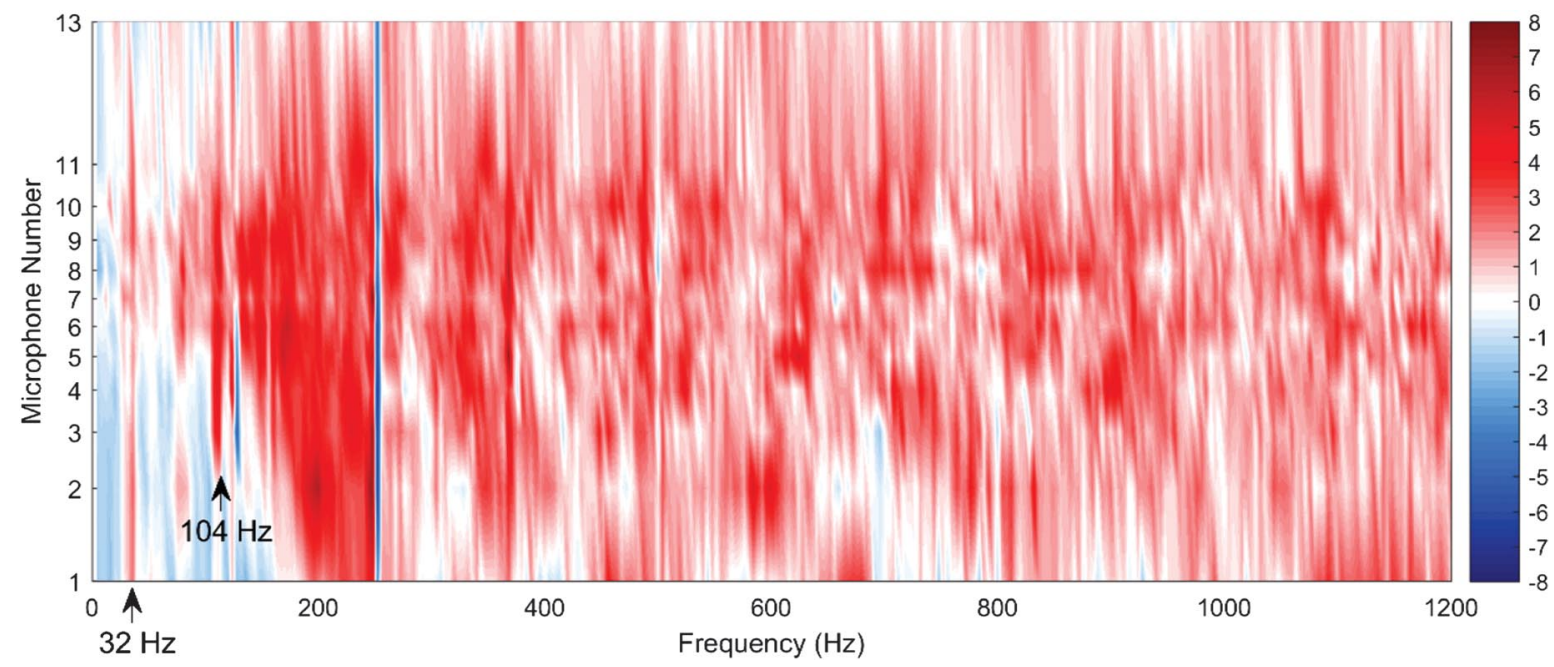

Fig. 16 (NLG-DressWTSD)-(NF) A-weighted $\triangle$ SPL. Contribution from the main strut, drag stay, and wheel bay. $50 \mathrm{~m} / \mathrm{s}$.

(NLG-DressWTSD) configuration, i.e., the main strut and drag stay in situ in the bay (see Fig. 6d), from which the closed bay fuselage (NF) configuration is subtracted. This results in the noise output from the main strut, drag stay, and bay only without the doors. In the figure, we see that the first duct tone is still clearly measurable as is an attenuated Helmholtz resonance. This result is highly significant. As we have seen previously, the doors are responsible for appreciable noise generation. However, their removal, although being a seemingly obvious step toward nose landing-gear noise reduction, might actually result in wheel bay cavity noise radiation, which to date has not been considered to be an issue.

\section{Attenuation of Landing-Gear Noise Through the Addition of Low-Noise Treatments}

The component removal assessment exercise presented previously was one part of the ALLEGRA nose landing-gear test campaign. As 
discussed, one of the principal objectives of the overall program was to assess the efficacy of high test readiness level low-noise treatments. Suitable technologies were downselected, and with the input of one of the ALLEGRA partners, Magnaghi, who is a landinggear manufacturer, the technologies were designed and built for testing, taking practical implementation issues into consideration. Such issues included the identification of appropriate attachment points on the landing gear, the potential for the physical interference of parts due to the kinematics as the landing gear folds away into the bay for stowage, and the deformation of the wheel tire upon touchdown of the aircraft. In addition, consideration of unexpected instability results, such as shimmy, due to the addition these add-ons needed to be considered [39].

This test campaign was quite extensive and requires a separate publication to disseminate the findings. However, a single technology is presented here to demonstrate how the preceding conclusions can be used to develop and validate noise reduction technologies.

Figure 17 shows the wheels of the NLG with and without hub caps applied. Because the nose landing gear does not require a braking system, the hub caps can be applied to the inside of the wheel. The photo of Fig. 17a is also useful to present because it allows the reader to see the details of the actual rim as manufactured.

Figure 18 presents again the NLG and the NLG-DressW configurations, this time for three flow velocities. Spectra for a flyover microphone position immediately above the wheels (Fig. 18a) as well as for a far-field microphone position chosen to examine the sideline radiation downstream (Fig. 18b) are presented. The noise generated by the interwheel rim modes at $636 \mathrm{~Hz}$ related to $l_{\mathrm{FF}}$ and $968 \mathrm{~Hz}$ related to $l_{\mathrm{EE}}$, as calculated as per the theory in Casalino et al. [32], are clearly visible and are constant in frequency for changing flow speed, supporting the hypothesis that they are governed by cavity resonance. Perhaps as to be expected, the lower-frequency energy at $636 \mathrm{~Hz}$ is more visible at the flyover position being generated within the wheels, closer to the axle, whereas the tone at $968 \mathrm{~Hz}$, related to the intertire gap, is more clearly visible at a downstream sideline position. The figure shows how these narrow bands disappear upon removal of the wheels: the NLG-DressW configuration.

The final plots in Fig. 19 show the benefit of applying the hub caps and compare the results to the configuration where the wheels are removed: NLG-DressW. Interestingly, for the flyover situation, the results are almost identical, indicating that most of the noise radiated as a result of the addition of the wheels can be attenuated with the addition of hub caps. In this flyover plot, Fig. 19a shows that, similar to removing the wheels, the addition of the hub caps reduces noise radiated to the flyover location by approximately $2-3 \mathrm{~dB}$ from $220 \mathrm{~Hz}$ to $1.25 \mathrm{kHz}$. Also, the hub caps successfully attenuate the rim mode at $636 \mathrm{~Hz}$. The hub caps also dramatically attenuate noise at $350 \mathrm{~Hz}$. No immediate explanation is available for this other than to

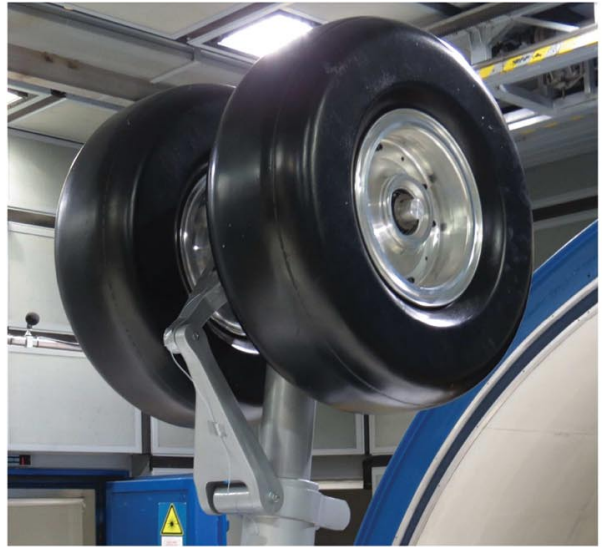

a) Without hub-caps applied

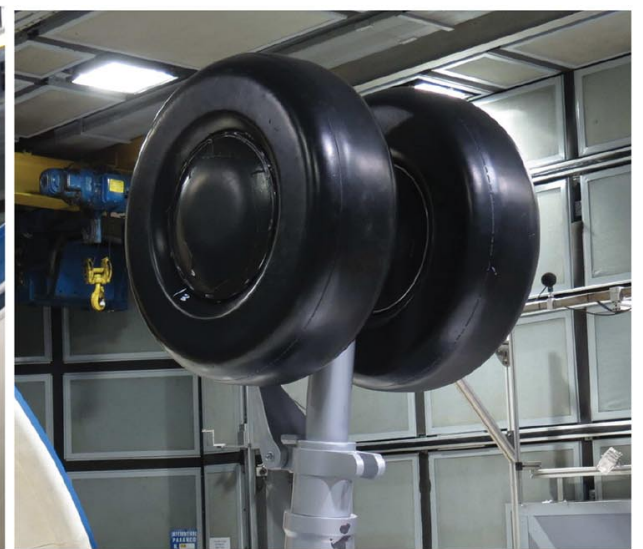

b) With hub caps applied

Fig. 17 Hub caps low-noise technology applied to nose landing gear.

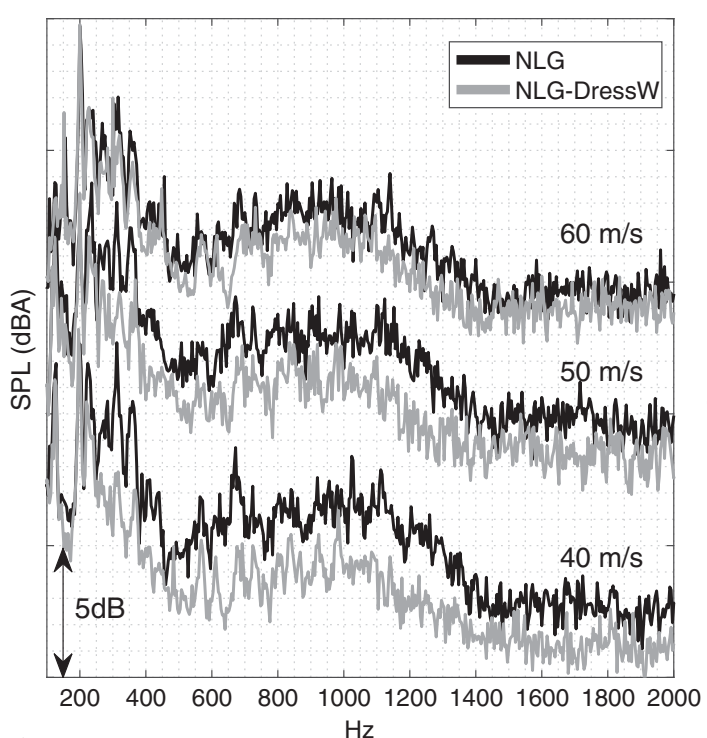

a) Flyover

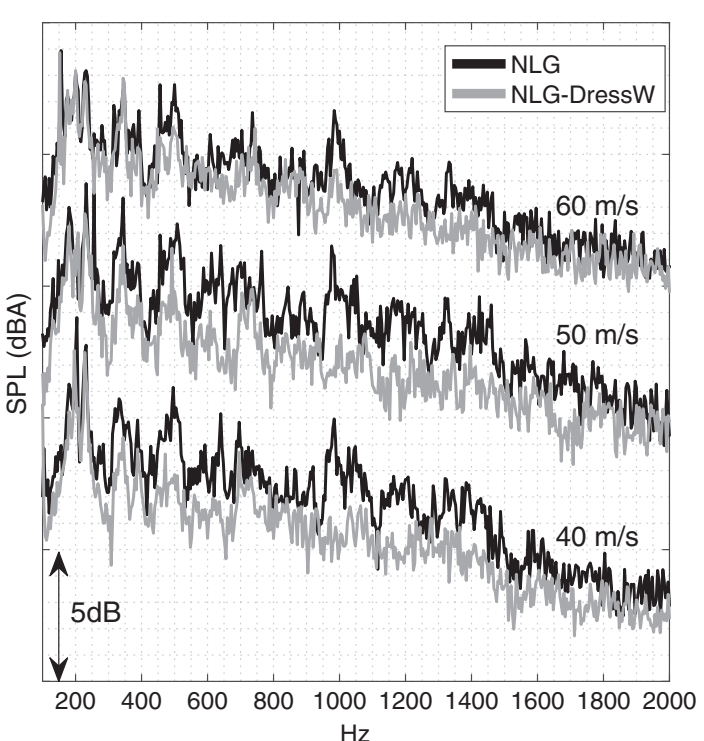

b) Sideline. Mic. 11

Fig. 18 Noise emission of NLG with and without wheels as a function of flow speed. Flyover and mic $11.50 \mathrm{~m} / \mathrm{s}$. 


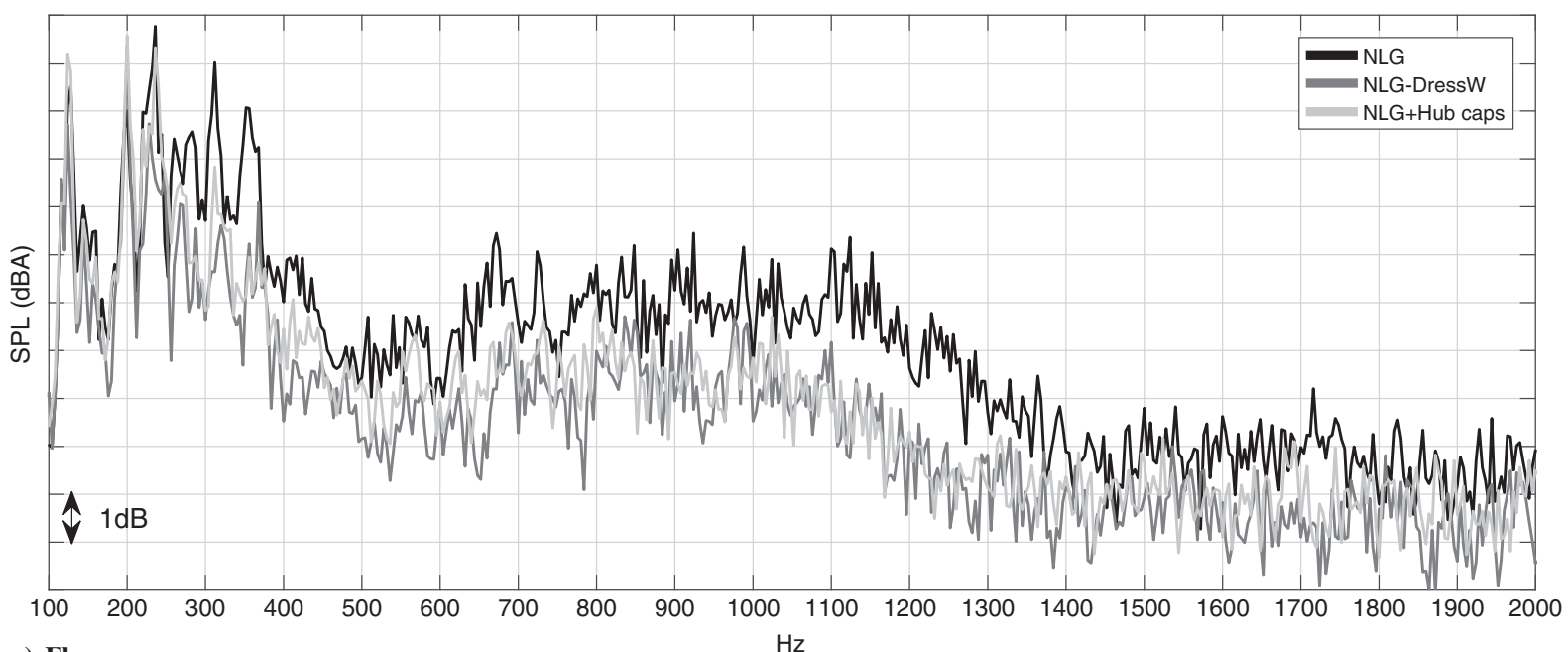

a) Flyover

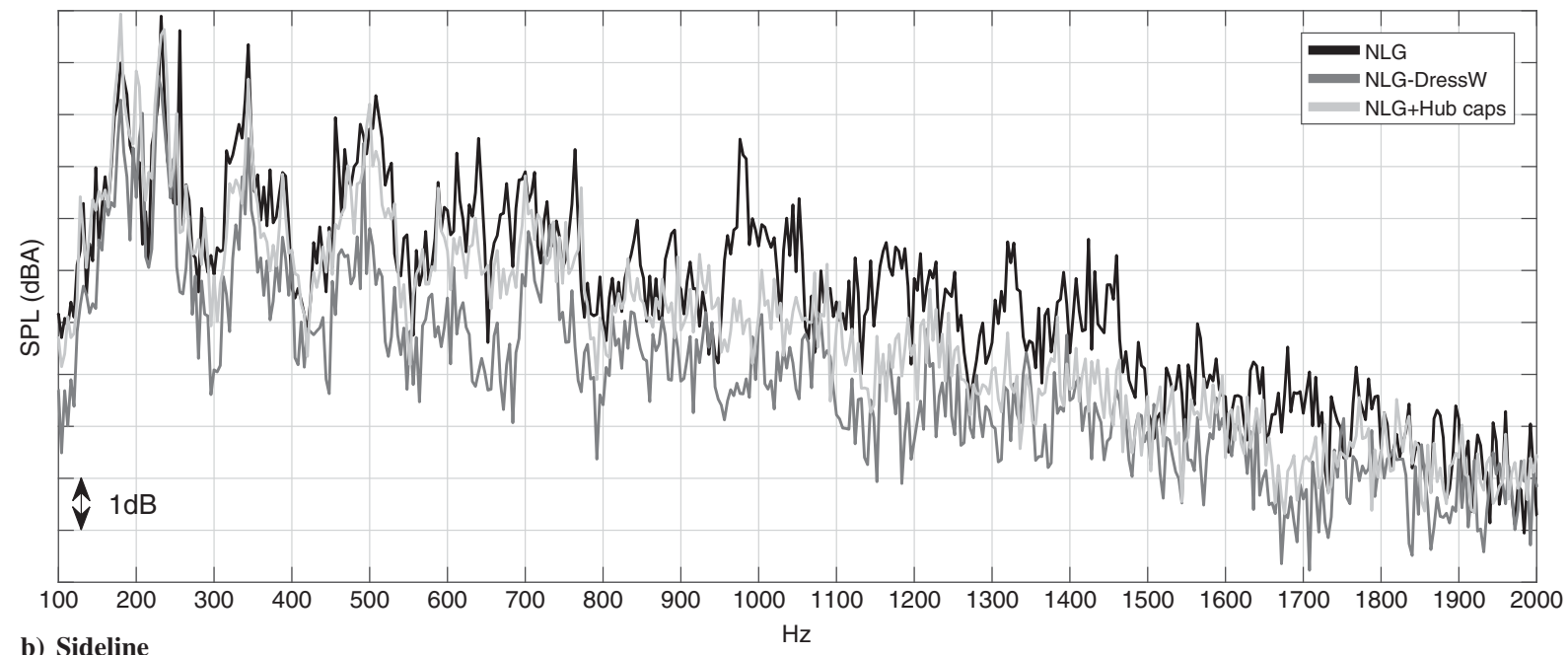

Fig. 19 Noise emission of NLG with wheel but no hub caps (NLG), without wheels (NLG-DressW), and with wheels and hub caps (NLG + Hub-caps). Flyover and mic $11.50 \mathrm{~m} / \mathrm{s}$.

observe that it is closely related to twice the wavelength of the $636 \mathrm{~Hz}$ tone. Further analysis of this and of potential azimuthal rim modes is required.

Figure $19 \mathrm{~b}$ presents the same results for microphone 11 of the farfield linear array. Here, we see the hub caps have successfully attenuated the $968 \mathrm{~Hz}$ rim mode. Again, there is attenuation at $350 \mathrm{~Hz}$, but unlike for the flyover location, the hub caps only seem to take effect from $900 \mathrm{~Hz}$, providing the same attenuation from this frequency as removing the wheels entirely. Below that frequency, it seems that the presence of the wheels generates noise that cannot be removed with the simple addition of the hub caps.

\section{Conclusions}

This paper presents experimental results from the Clean Sky, European Union-funded ALLEGRA "Advanced Low Noise Landing (Main and Nose) Gear for Regional Aircraft" collaborative research project. The deliverables were developed to assess low-noise technologies applied to a full-scale nose landing-gear model and a half-scale main landing-gear model of a 90-seat configuration regional aircraft concept. This Green Regional Aircraft concept was developed as an integrated technology demonstrator (ITD) to allow technologies that best suit the environmental goals set for regional aircraft, which will enter service from 2020 onward, to be matured and validated.

With regard to the nose landing-gear test campaign, one of the significant contributions of ALLEGRA is that a complete and highly detailed representation of the landing-gear components and associated structures such as the complete wheel bay cavity (wheel well), bay doors, nose fuselage, and hydraulic dressings were included at full scale. The model was designed and built by the consortium, with CAD input from the ITD topic manager LEONARDO, and tested in a large, aeroacoustic, open-jet, closedcircuit wind tunnel. To the authors' knowledge, this is the first time that a test program with these parameters, at full scale, has been examined in an experimental campaign.

This paper has focused on one element of the NLG testing: that of component removal assessment. The dressings, wheels, torque link, steering pinion, bay doors, and then the main strut and drag stay were removed in succession, and an acoustic evaluation was performed at a range of velocities from $M=0.12$ to $M=0.19$. In addition, one of the NLG low-noise technologies (the hub caps) was presented and assessed for efficacy.

Initially, the radiated noise from the entire model was assessed as a function of velocity. Two test configurations were initially compared: NF and NLG. NF was the background noise level baseline, which consisted of the nose fuselage with the landing gear removed and the wheel bay cavity sealed. The NF background noise output included the aerodynamic noise generated by the sealed fuselage within the wind tunnel in addition to the wind-tunnel noise itself. The NLG configuration consisted of the nose landing gear inserted into the wheel bay with the addition of bay doors. Thus, the NLG noise output included the noise generated by the gear, doors, wheel bay, fuselage, and any interactions between them, in addition to the wind-tunnel noise itself. From examination of the NLG and NF noise outputs in $1 / 3$ octave band, it was observed, for all velocities tested, that there 
was appreciable noise generated above the background noise level baseline up to the $10 \mathrm{kHz}$ center frequency. Comparing NLG to NF, as a function of narrowband frequency, it was found that the landing gear, doors, and bay generated up to $10 \mathrm{~dB}$ above the NF level between $120 \mathrm{~Hz}$ and approximately $1.2 \mathrm{kHz}$, with the greatest amount of noise, up to $12 \mathrm{~dB}$, generated in the frequency range between 120 and $400 \mathrm{~Hz}$. There was a bias to radiate upstream for high-amplitude noise centered at $200 \mathrm{~Hz}$, which was believed to be generated by the shear layer impinging on the drag stay and main strut, with contributions in this range from the doors and the steering pinion also. Given the high level of contribution from these components, deployable bay spoilers, such as examined by Dobrynski et al. [9] in the RAIN project and Neri et al. [40] in the ALLEGRA project, should be given serious consideration for production solutions. The pod gear concepts currently being investigated by NASA also offer great promise to attenuate these noise sources [41].

With the exception of some low-frequency tones, the sound pressure level (SPL) was found to scale with $V^{6}$ for low to midfrequencies up to $1.2 \mathrm{kHz}$. As discussed by Guo et al., it is believed, given the precise level of detail of the manufactured ALLEGRA NLG, that high-frequency noise should be radiated from the turbulent flow, which surrounds the small features of the model such as the hinges, wheel hub detail, fixtures, rods, ribs, pins, gussets, etc. For these small details, the compact requirement for the fluctuating pressure force dipole analogy theory no longer holds. As such, when scaled instead to $V^{7}$, above $1.2 \mathrm{kHz}$ up to $10 \mathrm{kHz}$, an excellent collapse of the sound pressure level curves was found.

Landing gear can consist of a large number of components clustered together of very different sizes, shapes, and orientations. Airflow over these components causes aerodynamic and tonal cavity noise, and depending on their position relative to each other, the total noise output of the assembly differs from that of the sum of the components in isolation due to installation effects. The research in this paper studied these effects and the influence of the components on each other by removing one component at a time and by subtracting the noise level of the assembly after from before the component was removed. This resulted in a measure equivalent to the effect of adding the component, which was examined for both noise reduction as well as noise addition. Noise reductions allow the installation effect of an added component to be understood.

Conclusions from this study showed that the addition of the doors and the main strut suppressed high-amplitude wheel bay resonant tones from radiating to the far field. The doors fully suppressed the first two wheel bay duct mode resonances at 104 and $160 \mathrm{~Hz}$ as well as the wheel bay Helmholtz resonance at $32 \mathrm{~Hz}$. The main strut and drag stay disturbed the shear layer enough to attenuate but not fully suppress the wheel bay Helmholtz resonance at $32 \mathrm{~Hz}$. All three of these tones could be excited within the velocity range of a landing aircraft, and thus it is important to take these mechanisms into account when implementing future design or operational changes. Bay spoilers and pod designs would also help here.

In addition to the main strut and drag stay attenuating wheel bay noise, a noise reduction, their installation in the empty bay also generated significant noise as a result of their presence in the mean flow and due to the wheel bay shear layer impinging upon them. Noise levels of up to $8 \mathrm{~dB}$ from $120 \mathrm{~Hz}$ to $1.2 \mathrm{kHz}$ result. The majority of the noise is located in the band between 120 to $300 \mathrm{~Hz}$, and through comparison with the overall noise generation NLG-NF, the main strut and drag stay contribute a significant and identifiable proportion in this frequency range, much of which radiates upstream. Vortex shedding at $148 \mathrm{~Hz}$ is measured from the main strut and increases in amplitude with the addition of the doors, torque link, and wheels.

The addition of the wheels also results in a significant and identifiable proportion to the overall NLG-NF level, up to $8 \mathrm{~dB}$ in the 260 to $400 \mathrm{~Hz}$ range but also at around $650 \mathrm{~Hz}$ and $1 \mathrm{kHz}$. These latter frequencies have been shown to correspond to wheel-to-wheel transversal acoustic modes and agree very well with previous work in the literature. The $650 \mathrm{~Hz}$ mode occurs due to a standing wave forming between the bases of the wheel rims and radiates more in the flyover direction, whereas the $1 \mathrm{kHz}$ mode seems to form between the wheel tires, causing lateral fluctuations clearly detectable at microphone positions located downstream along the sideline. The installation of the wheels seems to suppress vortex shedding at $200 \mathrm{~Hz}$ from the steering pinion.

As mentioned, the steering pinion generates narrow-band noise of $4 \mathrm{~dB}$ at its vortex shedding frequency of $200 \mathrm{~Hz}$ but also at approximately $320 \mathrm{~Hz}$, which is not well understood. The wheels tend to suppress the $200 \mathrm{~Hz}$ noise, but given the unsteadiness of the flow over the landing gear at approach conditions, this is not a reliable solution.

The aerodynamically unrefined doors, as well as suppressing wheel bay cavity noise, contribute significant levels of noise, up to $9 \mathrm{~dB}$, over a wide frequency range. These noise levels, as for all the components, should be assessed as a function of yaw angle, which could result in significant noise level increases.

The preceding analysis should be helpful for informing and validating semi-empirical and numerical methods and in the design of low-noise treatments. Results from the application of one such add-on low-noise treatment (wheel hub caps) was presented. The addition of the hub caps resulted in a reduction in noise level at the 90 deg fly over position equivalent to the removal of the wheels, which seems to suggest that, with the addition of the hub caps, all wheel noise is removed for that location. The $650 \mathrm{~Hz}$ wheel rim mode was successfully suppressed. For the sideline position, the addition of the hub caps also successfully suppressed the lateral wheel-to-wheel transversal mode at $1 \mathrm{kHz}$ and results in a noise reduction above $920 \mathrm{~Hz}$, equivalent to the removal of the wheels. For frequencies below that, little noise is attenuated when compared to the complete removal of the wheels, and thus an additional or different type of lownoise treatment would be required to address that frequency range.

\section{Acknowledgments}

The research leading to these results has received funding from the European Union's Seventh Framework Programme (FP7/20072013) for the Clean Sky Joint Technology Initiative under grant agreement 308225 (ALLEGRA). The authors acknowledge all the partners that took part in the ALLEGRA project: KTH Sweden, Pininfarina SPA, Eurotech, Teknosud, and Magnaghi Aeronautica, with TCD as coordinator. The authors would like to acknowledge the work of Francesco Amoroso of Eurotech, who led the manufacture of the model, Marco Esposito of Teknosud, who led the wind-tunnel model design, and Antonello Bianco for leading the wind-tunnel test campaign. The authors also acknowledge Michele Averardo and Massimiliano Di Giulio from LEONARDO Aircraft as Clean Sky topic managers.

\section{References}

[1] Black, D. A., Black, J. A., Issarayangyun, T., and Samuels, S. E., "Aircraft Noise Exposure and Resident's Stress and Hypertension: A Public Health Perspective for Airport Environmental Management," Journal of Air Transport Management, Vol. 13, No. 5, 2007, pp. 264-276. doi:10.1016/j.jairtraman.2007.04.003

[2] Lawton, R. N., and Fujiwara, D., "Living with Aircraft Noise: Airport Proximity, Aviation Noise and Subjective Wellbeing in England," Transportation Research Part D: Transport and Environment, Vol. 42, Jan. 2016, pp. 104-118.

doi:10.1016/j.trd.2015.11.002

[3] Astley, R. J., Agarwal, A., Joseph, P. F., Self, R. H., Smith, M. G., Sugimoto, R., and Tester, B. J., "Predicting and Reducing Aircraft Noise," Proceedings of the 14th International Congress on Sound Vibration, International Institute of Acoustics and Vibration (IIAV), Paper 819, 2007.

[4] Manoha, E., Sanders, L., and De La Puente, F., "Landing Gear Noise Prediction: What Is the Best Method?" Proceedings of 19th CEAS-ASC Workshop on Broadband Noise of Rotors and Airframe, The Aeroacoustics Specialists Committee of the Council of European Aerospace Societies, La Rochelle, France, 2015.

[5] Curle, N., "The Influence of Solid Boundaries upon Aerodynamic Sound," Proceedings of the Royal Society of London. Series A, Vol. 231, No. 1187, 1955, pp. 505-514. doi:10.1098/rspa.1955.0191

[6] Heller, H. H., and Dobrzynski, W. M., "Sound Radiation from Aircraft Wheel-Well/Landing-Gear Configurations," Journal of Aircraft, 
Vol. 14, No. 8, 1977, pp. 768-774. doi: $10.2514 / 3.58851$

[7] Dobrzynski, W., Buchholz, H., Dobrzynski, W., and Buchholz, H., "Full-Scale Noise Testing on Airbus Landing Gears in the German Dutch Wind Tunnel," 3rd AIAA/CEAS Aeroacoustics Conference, AIAA Paper 1997-1597, 1997. doi:10.2514/6.1997-1597

[8] Jaeger, S., Burnside, N., Soderman, P., Horne, W., and James, K., "Microphone Array Assessment of an Isolated, 26\%-Scale, HighFidelity Landing Gear," 8th AIAA/CEAS Aeroacoustics Conference \& Exhibit, AIAA Paper 2002-2410, June 2002. doi: $10.2514 / 6.2002-2410$

[9] Dobrzynski, W., Chow, L., Guion, P., and Shiells, D., "A European Study on Landing Gear Airframe Noise Sources," 6th Aeroacoustics Conference and Exhibit, AIAA Paper 2000-1971, June 2000. doi:10.2514/6.2000-1971

[10] Dobrzynski, W., "Almost 40 Years of Airframe Noise Research: What Did We Achieve?" Journal of Aircraft, Vol. 47, No. 2, 2010, pp. 353-367. doi:10.2514/1.44457

[11] Piet, J.F., Davy, R., Elias, G., Siller, H., Chow, L., Seror, C., and Laporte, F., "Flight Test Investigation of Add-On Treatments to Reduce Aircraft Airframe Noise," 11th AIAA/CEAS Aeroacoustics Conference, AIAA Paper 2005-3007, May 2005. doi:10.2514/6.2005-3007

[12] Pott-Pollenske, M., Dobrzynski, W., Buchholz, H., Guérin, S. Saueressig, G., and Finke, U., "Airframe Noise Characteristics from Flyover Measurements and Prediction," 12th AIAA/CEAS Aeroacoustics Conference, AIAA Paper 2006-2567, May 2006. doi:10.2514/6.2006-2567

[13] Dedoussi, I. C., Hynes, T. P., and Siller, H. A., "Investigating Landing Gear Noise Using Fly-Over Data: The Case of a Boeing 747-400," 19th AIAA/CEAS Aeroacoustics Conference, AIAA Paper 2013-2115, 2013. doi:10.2514/6.2013-2115

[14] Merino Martinez, R., Neri, E., Snellen, M., Kennedy, J., Simons, D., and Bennett, G. J., "Comparing Flyover Noise Measurements to Full-Scale Nose Landing Gear Wind Tunnel Experiments for Regional Aircraft," 23rd AIAA/CEAS Aeroacoustics Conference, AIAA Paper 2017-3006, 2017. doi:10.2514/6.2017-3006

[15] Fink, M. R., "Airframe Noise Prediction Method," Federal Aviation Administration, TR FAA-RD-77-29, Washington, D.C., 1977.

[16] Smith, M., and Chow, L., "Prediction Method for Aerodynamic Noise from Aircraft Landing Gear," 4th AIAA/CEAS Aeroacoustics Conference, AIAA Paper 1998-2228, 1998. doi: $10.2514 / 6.1998-2228$

[17] Guo, Y., "A Statistical Model for Landing Gear Noise Prediction," Journal of Sound and Vibration, Vol. 282, No. 1, 2005, pp. 61-87. doi:10.1016/j.jsv.2004.02.021

[18] Guo, Y., Yamamoto, K., and Stoker, R., "An Empirical Model for Landing Gear Noise Prediction," 10th AIAA/CEAS Aeroacoustics Conference, AIAA Paper 2004-2888, 2004. doi: $10.2514 / 6.2004-2888$

[19] Lopes, L. V., Brentner, K. S., and Morris, P. J., "Framework for a Landing-Gear Model and Acoustic Prediction," Journal of Aircraft, Vol. 47, No. 3, 2010, pp. 763-774 doi:10.2514/1.36925

[20] Hedges, L. S., Travin, A. K., and Spalart, P. R., "Detached-Eddy Simulations over a Simplified Landing Gear," Journal of Fluids Engineering, Vol. 124, No. 2, 2002, pp. 413-423. doi:10.1115/1.1471532

[21] Williams, J. E. F., and Hawkings, D. L., "Sound Generation by Turbulence and Surfaces in Arbitrary Motion," Proceedings of the Royal Society of London. Series A, Vol. 264, No. 1151, 1969, pp. 321-342. doi:10.1098/rsta.1969.0031

[22] Dahan, J., Futrzynski, R., O'Reilly, C., and Efraimsson, G., "AeroAcoustic Source Analysis of Landing Gear Noise via Dynamic Mode Decomposition," DIVA, Vol. 2, 2014, pp. 1245-1252.

[23] Dahan, J., O'Reilly, C., and Efraimsson, G., "Numerical Investigation of a Realistic Nose Landing Gear," 20th AIAA/CEAS Aeroacoustics Conference, AIAA Paper 2014-2077, 2014. doi:10.2514/6.2014-2077

[24] Casalino, D., Ribeiro, A. F. P., Fares, E., and Nölting, S., "LatticeBoltzmann Aeroacoustic Analysis of the LAGOON Landing-Gear Configuration," AIAA Journal, Vol. 52, No. 6, 2014, pp. 1232-1248. doi:10.2514/1.J052365

[25] Manoha, E., and Caruelle, B., "Summary of the LAGOON Solutions from the Benchmark Problems for Airframe Noise Computations-III Workshop," 21st AIAA/CEAS Aeroacoustics Conference, AIAA Paper
2015-2846, 2015

doi: $10.2514 / 6.2015-2846$

[26] Dobrzynski, W. M., Schöning, B., Chow, L. C., Wood, C., Smith, M., and Seror, C., "Design and Testing of Low Noise Landing Gears," International Journal of Aeroacoustics, Vol. 5, No. 3, 2006, pp. 233-262. doi:10.1260/1475-472X.5.3.233

[27] Guo, Y., Yamamoto, K. J., and Stoker, R. W., "Experimental Study on Aircraft Landing Gear Noise," Journal of Aircraft, Vol. 43, No. 2, 2006, pp. 306-317. doi: $10.2514 / 1.11085$

[28] Neri, E., Kennedy, J., and Bennett, G. J., "Bay Cavity Noise for FullScale Nose Landing Gear: A Comparison Between Experimental and Numerical Results," Aerospace Science and Technology, Vol. 72, Jan. 2018, pp. 278-291. doi:10.1016/j.ast.2017.11.016

[29] Ben Khelil, S., Bardoux, P., Godard, J.-L., Le Garrec, T., Kennedy, J., and Bennett, G. J., "Investigation of the Noise Emission of a Regional Aircraft Main Landing Gear Bay," 23rd AIAA/CEAS Aeroacoustics Conference, AIAA Paper 2017-3012, 2017. doi:10.2514/6.2017-3012

[30] Achenbach, E., and Heinecke, E., "On Vortex Shedding from Smooth and Rough Cylinders in the Range of Reynolds Numbers $6 \times 10^{3}$ to $6 \mathrm{e} 3$ to $5 \mathrm{e} 65 \times 10^{6}$," Journal of Fluid Mechanics, Vol. 109, Aug. 1981, pp. $239-251$. doi:10.1017/S002211208100102X

[31] Manoha, E., Bulté, J., and Caruelle, B., "Lagoon: An Experimental Database for the Validation of CFD/CAA Methods for Landing Gear Noise Prediction," 14th AIAA/CEAS Aeroacoustics Conference, AIAA Paper 2008-2816, May 2008. doi:10.2514/6.2008-2816

[32] Casalino, D., Ribeiro, A. F. P., and Fares, E., "Facing Rim Cavities Fluctuation Modes," Journal of Sound and Vibration, Vol. 333, No. 13, 2014, pp. 2812-2830. doi:10.1016/j.jsv.2014.01.028

[33] Bennett, G. J., Stephens, D. B., and Rodriguez Verdugo, F., "Resonant Mode Characterisation of a Cylindrical Helmholtz Cavity Excited by a Shear Layer," Journal of the Acoustical Society of America, Vol. 141, No. 1, 2017, pp. 7-18. doi:10.1121/1.4973212

[34] Stephens, D. B., Verdugo, F. R., and Bennett, G. J., "Shear Layer Driven Acoustic Modes in a Cylindrical Cavity," Journal of Pressure Vessel Technology, Vol. 136, No. 5, 2014, Paper 051309. doi:10.1115/1.4026866

[35] Bennett, G. J., Rodriguez Verdugo, F., and Stephens, D. B., "Shear Layer Dynamics of a Cylindrical Cavity for Different Acoustic Resonance Modes," 15th International Symposium on Applications of Laser Techniques to Fluid Mechanics, Instituto Superior Técnico Paper 1727, Lisbon, Portugal, July 2010, http://www.tara.tcd.ie/handle/2262/ 41052

[36] Marsden, O., Jondeau, E., Souchotte, P., Bogey, C., Bailly, C., and Juvé, D., "Investigation of Flow Features and Acoustic Radiation of a Round Cavity," 14th AIAA/CEAS Aeroacoustics Conference, AIAA Paper 2008-2851, May 2008. doi: $10.2514 / 6.2008-2851$

[37] Bennett, G. J., and Fitzpatrick, J. A., "Noise-Source Identification for Ducted Fan Systems," AIAA Journal, Vol. 46, No. 7, 2008, pp. 1663 1674 . doi: $10.2514 / 1.33522$

[38] Davis, I., and Bennett, G. J., "Spectral, Modal and Coherence Analysis of Sum and Difference Scattering of Narrowband Noise in Turbomachinery," International Journal of Aeroacoustics, Vol. 15, Nos. 1-2, 2016, pp. 180-206. doi: $10.1177 / 1475472 X 16630860$

[39] Eret, P., Kennedy, J., and Bennett, G. J., "Effect of Noise Reducing Components on Nose Landing Gear Stability for a Mid-Size Aircraft Coupled with Vortex Shedding and Freeplay," Journal of Sound and Vibration, Vol. 354, Oct. 2015, pp. 91-103. doi:10.1016/j.jsv.2015.06.022

[40] Neri, E., Kennedy, J., and Bennett, G. J., "Characterization of Low Noise Technologies Applied to a Full Scale Fuselage Mounted Nose Landing Gear," ASME 2015 Noise Control and Acoustics Division Conference, San Francisco, CA, 2015, p. V001T01A005. doi:10.1115/NCAD2015-5911

[41] Thomas, R. H., Guo, Y., Berton, J., and Fernandez, H., "Aircraft Noise Reduction Technology Roadmap Toward Achieving the NASA 2035 Goal," 23rd AIAA/CEAS Aeroacoustics Conference, AIAA Paper 20173193, 2017.

doi:10.2514/6.2017-3193 